FIAN/TD-10/10

ITEP/TH-44/10

\title{
Towards a proof of AGT conjecture by methods of matrix models
}

\author{
A.Mironov $\$$ A.Morozov ${ }^{\Uparrow}$ and Sh.Shakirov" ${ }^{\|}$
}

\begin{abstract}
A matrix model approach to proof of the AGT relation is briefly reviewed. It starts from the substitution of conformal blocks by the Dotsenko-Fateev $\beta$-ensemble averages and Nekrasov functions by a double deformation of the exponentiated Seiberg-Witten prepotential in $\beta \neq 1$ and $g_{s} \neq 0$ directions. Establishing the equality of these two quantities is a typical matrix model problem, and it presumably can be ascertained by investigation of integrability properties and developing an associated Harer-Zagier technique for evaluation of the exact resolvent.
\end{abstract}

\section{Introduction}

The AGT relation [1] experimentally discovered about 1.5 years ago, brings together a number of different subjects some of them listed in Fig.1. In this short review we are going to explain them very briefly. The details and various discussions of the AGT conjecture can be found in [2]-[25]. The correspondence shown in the figure, can be described at different levels: as representation theory, as in the first line; as a direct equality of two quantities in the second line: conformal blocks of two-dimensional conformal theory and Nekrasov functions of SeibergWitten (SW) theory; and as a relation between matrix models and Seiberg-Witten theory in the third line. The main problem with the first two options is the lack of a reasonable conceptual definition of the Nekrasov functions, only operational technical definitions are available so far. At the third level, the Nekrasov functions are considered as providing a double-deformation with the help of the two $\epsilon$-parameters of the Seiberg-Witten theory. We find this point of view at the AGT conjecture the most interesting and important. It involves, at the physical level, the correspondence between gauge theories and integrable systems, which are well known to stand behind both the matrix models [26]-[29] and the SW theory [30, 31, 32]. In this paper we rely upon the standard dictionary (gauge theory $\leftrightarrow$ integrable system) [31]. Also we use the term "matrix models" in a wider sense: the eigenvalue $\beta$-ensembles. All the definitions from $2 d$ conformal theory used below can be found in [33] and [3].

In a few first sections we remind the definitions of the quantities that are directly used in the AGT correspondence: the conformal blocks and Nekrasov functions, and formulate the AGT conjecture. Then we explain what definition of SW theory and of matrix models we use. After this, we are already able to formulate the AGT correspondence as a matrix models/SW theory correspondence in a more concrete form (figures 5 and 6 ). The rest of the paper is devoted to the discussion of its details.

At the end of the paper we add three Appendices. Two of them are aimed to illustrate the non-widely-known chapters of matrix model theory, which are heavily exploited in the main

\footnotetext{
${ }^{\S}$ Lebedev Physics Institute and ITEP, Moscow, Russia; mironov@itep.ru; mironov@lpi.ru

"ITEP, Moscow, Russia and Laboratoire de Mathematiques et Physique Theorique, CNRS-UMR 6083, Universite Francois Rabelais de Tours, France; morozov@itep.ru

"ITEP, Moscow, Russia and MIPT, Dolgoprudny, Russia; shakirov@itep.ru
} 
rep.theory of

Virasoro algebra

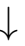

$2 d$

hypergeometric $\stackrel{c=\infty}{\longleftarrow}$

series conformal

blocks
.

2

(1) rep.theor

of $S_{\infty}$

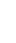

$+2$

$\stackrel{A \underline{\underline{G}}}{=}$

$4 d$

Nekrasov $\longleftarrow$

LMNS

functions

integrals

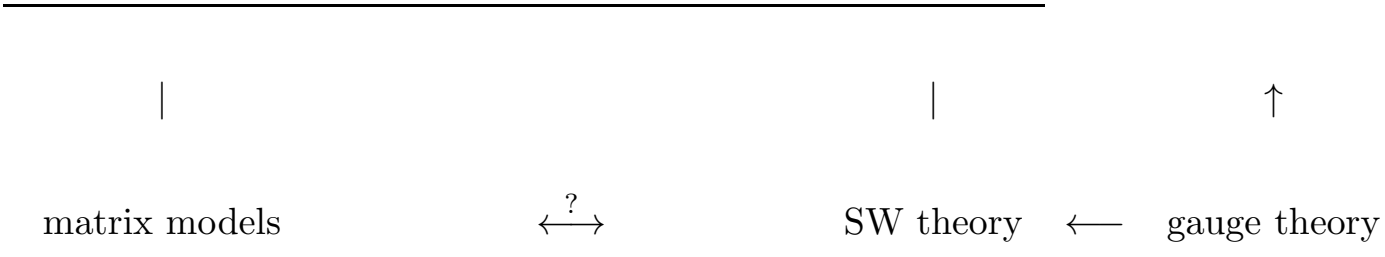

integrable systems

Figure 1: Interrelations between various fields touched by the AGT conjecture.

text. The first of them is the Harer-Zagier explicit formula for the exact (all genus) one-point resolvent $\rho_{1}$, which is not directly deducible from the Virasoro constraints and, thus, from the $\mathrm{AMM} / \mathrm{EO}$ version of topological recursion; rather it is a direct corollary of a hidden integrable structure of the free energy. The second one is the SW representation of the $\beta$-ensemble free energy, $\delta F=\beta \sum_{I} \oint_{B_{I}} \rho_{1} \delta \oint_{A_{I}} \rho_{1}$ through contour integrals of the exact one-point resolvent. We illustrate both subjects with the simplest example of the Gaussian phase of the Hermitian matrix model with $\beta=1$. The program outlined in the present paper, requires an extension of this example to the Dotsenko-Fateev (DF) $\beta$-ensemble. The third Appendix contains a brief description of various limits of the AGT relation, including the highly non-trivial "pure gauge" and "stationary" $\left(\epsilon_{2} \rightarrow 0\right)$ limits.

\section{Conformal blocks}

The 4-point conformal block is a function of the double ratio of 4 points on the Riemann sphere, $q=\frac{\left(z_{1}-z_{2}\right)\left(z_{3}-z_{4}\right)}{\left(z_{1}-z_{3}\right)\left(z_{2}-z_{4}\right)}$ which is parameterized by four external and one intermediate conformal dimensions $\Delta_{i}(i=1,2,3,4), \Delta$ and the central charge $c$ of conformal theory [33. The conformal block is completely fixed by the conformal properties and, at small enough $q$, it can be expanded into the power series:

$$
\mathcal{B}_{\Delta}\left(\Delta_{1}, \Delta_{2} ; \Delta_{3}, \Delta_{4} \mid q\right)=\sum_{n} q^{n} \mathcal{B}_{\Delta}^{(n)}
$$

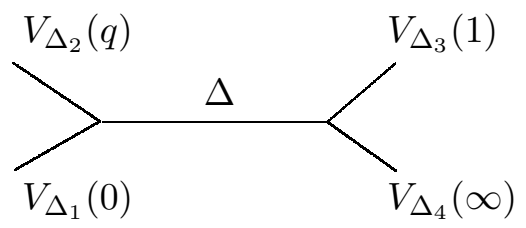


where the first coefficients of expansion are

$$
\begin{gathered}
\mathcal{B}_{\Delta}^{(0)}=1 \\
\mathcal{B}_{\Delta}^{(1)}=\frac{\left(\Delta+\Delta_{1}-\Delta_{2}\right)\left(\Delta+\Delta_{3}-\Delta_{4}\right)}{2 \Delta} \\
\mathcal{B}_{\Delta}^{(2)}=\frac{\left(\Delta+\Delta_{1}-\Delta_{2}\right)\left(\Delta+\Delta_{1}-\Delta_{2}+1\right)\left(\Delta+\Delta_{3}-\Delta_{4}\right)\left(\Delta+\Delta_{3}-\Delta_{4}+1\right)}{4 \Delta(2 \Delta+1)}+ \\
+\frac{\left[\left(\Delta_{2}+\Delta_{1}\right)(2 \Delta+1)+\Delta(\Delta-1)-3\left(\Delta_{2}-\Delta_{1}\right)^{2}\right]\left[\left(\Delta_{3}+\Delta_{4}\right)(2 \Delta+1)+\Delta(\Delta-1)-3\left(\Delta_{3}-\Delta_{4}\right)^{2}\right]}{2(2 \Delta+1)(2 \Delta(8 \Delta-5)+(2 \Delta+1) c)}
\end{gathered}
$$

These expressions become fast quite involved. However, at the particular case of large central charge $c \longrightarrow \infty$ the conformal block drastically simplifies becoming the hypergeometric function

$$
\mathcal{B}_{\Delta}\left(\Delta_{1}, \Delta_{2} ; \Delta_{3}, \Delta_{4} \mid q\right) \stackrel{c \longrightarrow \infty}{\longrightarrow} F\left(\Delta+\Delta_{1}-\Delta_{2}, \Delta+\Delta_{3}-\Delta_{4} ; 2 \Delta \mid q\right)
$$

\section{The origin of conformal blocks}

If one writes the conformal block as a correlator in a chiral algebra with a projector to the intermediate state inserted

$$
\mathcal{B}_{\Delta}\left(\Delta_{1}, \Delta_{2} ; \Delta_{3}, \Delta_{4} \mid q\right)=\left\langle V_{\Delta_{1}(0)} V_{\Delta_{2}}(q) P_{\Delta} V_{\Delta_{3}}(1) V_{\Delta_{4}}(\infty)\right\rangle
$$

its generic structure can be determined using the operator product expansion of two primary chiral vertices

$$
V_{\hat{\alpha}}(z) V_{\hat{\beta}}\left(z^{\prime}\right)=\sum_{\hat{\gamma}} \frac{\mathcal{C}_{\hat{\alpha} \hat{\beta}}^{\hat{\gamma}} V_{\hat{\gamma}}\left(z^{\prime}\right)}{\left(z-z^{\prime}\right)^{\Delta_{\hat{\alpha}}+\Delta_{\hat{\beta}}-\Delta_{\hat{\gamma}}}}
$$

of the Virasoro algebra

$$
\left[L_{m}, L_{n}\right]=(m-n) L_{m+n}+\frac{c}{12} n\left(n^{2}-1\right) \delta_{n+m, 0}
$$

The conformal dimension of the vertex is given by

$$
L_{0} V_{\alpha}=\Delta_{\alpha} V_{\alpha}
$$

and the descendant operators are defined by

$$
V_{\alpha, Y}=L_{-k_{l}} \ldots L_{-k_{2}} L_{-k_{1}} V_{\alpha}
$$

Then, the conformal block is manifestly given by the formula

$$
\begin{gathered}
\mathcal{B}_{\Delta}\left(\Delta_{1}, \Delta_{2} ; \Delta_{3}, \Delta_{4} \mid q\right)=\sum_{|Y|=\left|Y^{\prime}\right|} q^{|Y|} \mathcal{B}_{Y, Y^{\prime}}=\sum_{|Y|=\left|Y^{\prime}\right|} q^{|Y|} \beta_{\Delta_{1} \Delta_{2}}^{\Delta}(Y) Q_{\Delta}\left(Y, Y^{\prime}\right) \beta_{\Delta_{3} \Delta_{4}}^{\Delta}\left(Y^{\prime}\right) \\
=\sum_{|Y|=\left|Y^{\prime}\right|} q^{|Y|} \gamma_{\Delta_{1} \Delta_{2} \Delta}(Y) Q_{\Delta}^{-1}\left(Y, Y^{\prime}\right) \gamma_{\Delta_{3} \Delta_{4} \Delta}\left(Y^{\prime}\right)
\end{gathered}
$$


where the 3 -vertices are manifestly given by formulas

$$
\gamma_{\Delta_{1}, \Delta_{2} ; \Delta}(Y)=\left\langle L_{-Y} V_{\Delta}(0) V_{\Delta_{1}}(1) V_{\Delta_{2}}(\infty)\right\rangle=\prod_{i}\left(\Delta+k_{i} \Delta_{1}-\Delta_{2}+\sum_{j<i} k_{j}\right)
$$

and the Shapovalov matrix is defined as a scalar product

$$
Q_{\Delta}\left(Y, Y^{\prime}\right)=\left\langle L_{-Y} V_{\Delta}(0) L_{-Y^{\prime}} V_{\Delta}(\infty)\right\rangle
$$

\section{Nekrasov functions}

The Nekrasov functions for $\mathcal{N}=2$ SUSY $S U(2)$ gauge theory with four fundamental matter hypermultiplets (the $\beta$-function in such a theory is equal to zero) at first levels are manifestly given by formulas (see [34] for generic expressions)

$$
\begin{aligned}
& \mathcal{Z}_{[1][0]}=-\frac{1}{\epsilon_{1} \epsilon_{2}} \cdot \frac{\prod_{r=1}^{4}\left(a+\mu_{r}\right)}{2 a(2 a+\epsilon)} \\
& \mathcal{Z}_{[0][1]}=-\frac{1}{\epsilon_{1} \epsilon_{2}} \cdot \frac{\prod_{r=1}^{4}\left(a-\mu_{r}\right)}{2 a(2 a-\epsilon)} ; \\
& \mathcal{Z}_{[2][0]}=\frac{1}{2 ! \epsilon_{1} \epsilon_{2}^{2}\left(\epsilon_{1}-\epsilon_{2}\right)} \cdot \frac{\prod_{r=1}^{4}\left(a+\mu_{r}\right)\left(a+\mu_{r}+\epsilon_{2}\right)}{2 a\left(2 a+\epsilon_{2}\right)(2 a+\epsilon)\left(2 a+\epsilon+\epsilon_{2}\right)}, \\
& \mathcal{Z}_{[0][2]}=\frac{1}{2 ! \epsilon_{1} \epsilon_{2}^{2}\left(\epsilon_{1}-\epsilon_{2}\right)} \cdot \frac{\prod_{r=1}^{4}\left(a-\mu_{r}\right)\left(a-\mu_{r}-\epsilon_{2}\right)}{2 a\left(2 a-\epsilon_{2}\right)(2 a-\epsilon)\left(2 a-\epsilon-\epsilon_{2}\right)}, \\
& \mathcal{Z}_{[11][0]}=-\frac{1}{2 ! \epsilon_{1}^{2} \epsilon_{2}\left(\epsilon_{1}-\epsilon_{2}\right)} \cdot \frac{\prod_{r=1}^{4}\left(a+\mu_{r}\right)\left(a+\mu_{r}+\epsilon_{1}\right)}{2 a\left(2 a+\epsilon_{1}\right)(2 a+\epsilon)\left(2 a+\epsilon+\epsilon_{1}\right)}, \\
& \mathcal{Z}_{[0][11]}=-\frac{1}{2 ! \epsilon_{1}^{2} \epsilon_{2}\left(\epsilon_{1}-\epsilon_{2}\right)} \cdot \frac{\prod_{r=1}^{4}\left(a-\mu_{r}\right)\left(a-\mu_{r}-\epsilon_{1}\right)}{2 a\left(2 a-\epsilon_{1}\right)(2 a-\epsilon)\left(2 a-\epsilon-\epsilon_{1}\right)}, \\
& \mathcal{Z}_{[1][1]}=\frac{1}{\epsilon_{1}^{2} \epsilon_{2}^{2}} \cdot \frac{\prod_{r=1}^{4}\left(a+\mu_{r}\right)\left(a-\mu_{r}\right)}{\left(4 a^{2}-\epsilon_{1}^{2}\right)\left(4 a^{2}-\epsilon_{2}^{2}\right)} ; \\
& \mathcal{Z}_{[3][0]}=-\frac{1}{3 ! \epsilon_{1} \epsilon_{2}^{3}\left(\epsilon_{1}-\epsilon_{2}\right)\left(\epsilon_{1}-2 \epsilon_{2}\right)} \cdot \frac{\prod_{r=1}^{4}\left(a+\mu_{r}\right)\left(a+\mu_{r}+\epsilon_{2}\right)\left(a+\mu_{r}+2 \epsilon_{2}\right)}{2 a\left(2 a+\epsilon_{2}\right)\left(2 a+2 \epsilon_{2}\right)(2 a+\epsilon)\left(2 a+\epsilon+\epsilon_{2}\right)\left(2 a+\epsilon+2 \epsilon_{2}\right)},
\end{aligned}
$$

Here

$$
\epsilon=\epsilon_{1}+\epsilon_{2}
$$

\section{An origin of Nekrasov functions}

The origin of the Nekrasov functions is the LMNS integrals [35, which are integrals over a regularized moduli space of instantons, that is, the space of ADHM configurations. These integrals are manifestly given by formulas

$$
Z=e^{F}=\sum_{k} q^{k} \oint \prod_{a<b}^{k} \frac{\chi_{a b}^{2}\left(\chi_{a b}^{2}-\left(\epsilon_{1}-\epsilon_{2}\right)^{2}\right)}{\left(\chi_{a b}^{2}-\epsilon_{1}^{2}\right)\left(\chi_{a b}^{2}-\epsilon_{2}^{2}\right)} \prod_{a=1}^{k} \frac{P\left(\chi_{a}\right)}{Q\left(\chi_{a}\right)} d \chi_{a}=\sum_{\substack{\text { over pairs of } \\ \text { Young diagrams }}} Z_{N e k}\left(Y, Y^{\prime}\right)
$$




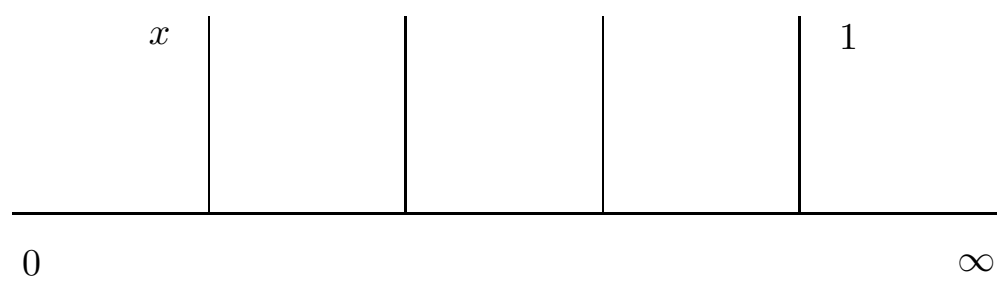

Figure 2: The diagram describing the multi-point conformal block of the "comb" type, which is involved into the AGT relation.

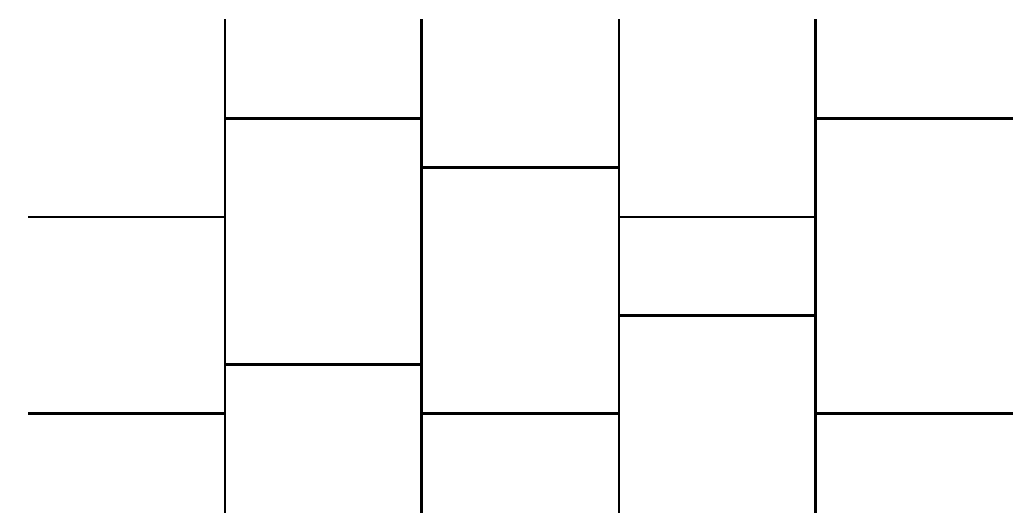

Figure 3: The brane diagram AGT related to the comb conformal block in Fig.2.

with $\chi_{a b}=\chi_{a}-\chi_{b}$ and the polynomials

$$
P(x)=\prod_{a=1}^{4}\left(x+\mu_{a}\right), \quad Q(x)=\left(x^{2}-a^{2}\right)\left((x+\epsilon)^{2}-a^{2}\right)
$$

depend on the vacuum expectation value $a$ of the scalar in the gauge theory and fundamental hypermultiplet masses $\mu_{a}$. These integrals can be manifestly taken and represented as sums over Young diagrams (integer partitions) 34].

The associated brane diagrams are the tropical limits of $2 d$ Riemann surfaces. The correspondence between the conformal blocks and the brane diagrams is drawn on figures 2 and 3.

Here we pictured the correspondence for the comb type diagrams. The other type of diagrams are obtained from this one by a transformation of S-duality, see Fig, 4. The elements of matrix of the same transformation at the level of representation theory (of Virasoro algebra) are named the Racah coefficients (while the conformal block is nothing but the Clebsch-Gordan coefficients, which depend on the parameter $q$, since in the Virasoro case the co-product is parameterized by this parameter [36]).

\section{$6 \quad$ AGT relation}

The AGT conjecture literally states that

$$
\begin{gathered}
\mathcal{B}_{\Delta}\left(\Delta_{1}, \Delta_{2} ; \Delta_{3}, \Delta_{4} \mid q\right)=\sum_{n} x^{n} \mathcal{B}_{\Delta}^{(n)}=\sum_{|Y|=\left|Y^{\prime}\right|} q^{|Y|} \mathcal{B}_{Y, Y^{\prime}}^{\alpha}\left(\alpha_{1}, \alpha_{2} ; \alpha_{3}, \alpha_{4}\right)= \\
=(1-q)^{-\nu} \sum_{Y, Y^{\prime}} q^{|Y|+\left|Y^{\prime}\right|} \mathcal{Z}_{Y, Y^{\prime}}^{S U(2)} \equiv \sum_{Y, Y^{\prime}} q^{|Y|+\left|Y^{\prime}\right|} \mathcal{Z}_{Y, Y^{\prime}}^{U(2)}
\end{gathered}
$$




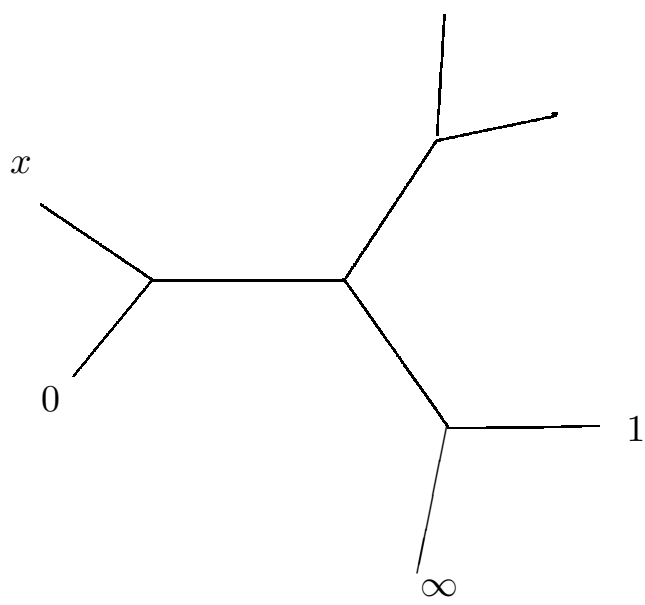

Figure 4: One of the diagrams obtained from the comb diagram of Fig.2 by an S-duality transformation.

where $\mathcal{Z}_{Y, Y^{\prime}}^{S U(2)}$ are given by the formulas of section 4 .

The simplest example of the conjecture comes from the first level (linear term in $q$ ):

$$
\begin{gathered}
\mathcal{B}_{\Delta}^{(1)}=\frac{\left(\Delta+\Delta_{1}-\Delta_{2}\right)\left(\Delta+\Delta_{3}-\Delta_{4}\right)}{2 \Delta}= \\
=\mathcal{Z}_{[1][0]}+\mathcal{Z}_{[0][1]}+\nu=-\frac{1}{\epsilon_{1} \epsilon_{2}} \cdot \frac{\prod_{r=1}^{4}\left(a+\mu_{r}\right)}{2 a(2 a+e)}-\frac{1}{\epsilon_{1} \epsilon_{2}} \cdot \frac{\prod_{r=1}^{4}\left(a-\mu_{r}\right)}{2 a(2 a-e)}+\nu
\end{gathered}
$$

which is correct provided that

$$
\begin{gathered}
\Delta_{i}=\frac{\alpha_{i}\left(\epsilon-\alpha_{i}\right)}{\epsilon_{1} \epsilon_{2}} \\
a+\frac{\epsilon}{2}=\alpha \\
\mu_{1}=-\frac{\epsilon}{2}+\alpha_{1}+\alpha_{2}, \quad \mu_{2}=\frac{\epsilon}{2}+\alpha_{1}-\alpha_{2}, \quad \mu_{3}=-\frac{\epsilon}{2}+\alpha_{3}+\alpha_{4}, \quad \mu_{4}=\frac{\epsilon}{2}+\alpha_{3}-\alpha_{4}, \\
\nu=\frac{2 \alpha_{1} \alpha_{3}}{\epsilon_{1} \epsilon_{2}}
\end{gathered}
$$

At higher levels one also needs

$$
c=1+\frac{6\left(\epsilon_{1}+\epsilon_{2}\right)^{2}}{\epsilon_{1} \epsilon_{2}}=1-6\left(b-\frac{1}{b}\right)^{2}
$$

with

$$
b=\sqrt{-\epsilon_{1} / \epsilon_{2}}, \quad g_{s}=\sqrt{-\epsilon_{1} \epsilon_{2}}
$$

\section{Seiberg-Witten representation of prepotential}

We call SW prepotential a function $\mathcal{F}\left(a_{1}, \ldots, a_{n}\right)$ of $n$ variables defined with the help of the SW data: a complex curve $\Sigma$ and an meromorphic 1-differential $\Omega$ on it, through the SW system of equations: 


$$
\left\{\begin{array}{c}
a_{I}=\oint_{A_{I}} \Omega \\
\frac{\partial \mathcal{F}}{\partial a_{I}}=\oint_{B_{I}} \Omega
\end{array}\right.
$$

In our study it appears in two contexts.

I. SW theory associated with the $S U(2)$ gauge model. The prepotential $\mathcal{F}_{0}^{S U(2)}(a)$ of a single variable $a$ is defined on $\Sigma^{S U(2)}$ of genus one and [37, (1.1)]

$$
\begin{gathered}
\Omega_{0}^{S U(2)}=p d \phi, \\
\Sigma^{S U(2)}: \quad p^{2}-P_{+}(p) e^{i \phi}-P_{-}(p) e^{-i \phi}=E
\end{gathered}
$$

where

$$
P_{+}(p-h)=\frac{\left(p-\mu_{1}\right)\left(p-\mu_{2}\right)}{(1+q)}, \quad P_{-}(p-h)=\frac{q\left(p-\mu_{3}\right)\left(p-\mu_{4}\right)}{(q+1)}, \quad h \equiv \frac{\sum_{i=1}^{4} \mu_{i}}{2(q+1)}
$$

and $q$ is a function of the coupling constant $\tau, q=\theta_{4}^{4} / \theta_{2}^{4}$ (see [37, 9]). This SW system corresponds to the XXX spin chain on 2 sites [31]. The limit of all four hypermultiplet masses infinite (see s,14) leads to the pure gauge theory on the physical side [38, to the periodic Toda chain on 2 sites on the integrable side [31] and to a degenerated conformal block on the conformal side [4]. In this case, the corresponding SW pair (21) is

$$
\begin{gathered}
\Omega_{0}^{S U(2), p g}=p d \phi \\
\Sigma^{S U(2), p g}: \quad \frac{1}{2} p^{2}-\Lambda^{2} \cos \phi=E
\end{gathered}
$$

The prepotential $\mathcal{F}_{0}^{S U(2)}(a)$ is lifted up to the Nekrasov function $\mathcal{F}^{S U(2)}(a)$ so that $\mathcal{F}^{S U(2)}(a) \rightarrow$ $\mathcal{F}_{0}^{S U(2)}(a)$ in the limit $\epsilon_{1}=-\epsilon_{2} \rightarrow 0$.

II. SW theory associated with the matrix model. In any eigenvalue matrix model one can define a resolvent and its genus expansion in powers of $g_{s}$ (or $1 / N$ ) [39]-[42] (see s.13 for accurate definitions, the sign minus in front of the SW differential is due to the sign minus in front of the matrix model potential, (48)):

$$
-\Omega^{(m m)}=\rho^{(m m)}(z)=\left\langle\operatorname{Tr} \frac{1}{z-M}\right\rangle_{(m m)}=\sum_{p=0}^{\infty} g_{s}^{2 p} \rho_{p}^{(m m)}(z)
$$

All $\rho_{p}^{(m m)}(z)$ are meromorphic 1-differentials on a spectral curve $\Sigma^{(m m)}$ and the free energy $\mathcal{F}^{(m m)}=\sum_{p=0}^{\infty} g_{s}^{2 p} \mathcal{F}_{p}^{(m m)}$ satisfies the SW equations, (20). We illustrate how formulas (20) work in the simplest case of the Gaussian matrix model in Appendix II.

In fact, along with the SW pair $\left(\mathcal{F}^{(m m)}, \rho^{(m m)}\right)$, one also can associate with each matrix model the "quasiclassical" (genus zero) SW pair $\left(\mathcal{F}_{0}^{(m m)}, \rho_{0}^{(m m)}\right)$.

As any partition function, the exponential of $\mathcal{F}^{(m m)}$ is a $\tau$-function, satisfying bilinear Hirota equations [43, while $\mathcal{F}_{0}^{(m m)}$ is a far more sophisticated "quasiclassical" $\tau$-function, satisfying a system of highly non-linear WDVV equations [44]

$$
F_{I} F_{J}^{-1} F_{K}=F_{K} F_{J}^{-1} F_{I}
$$

where $n \times n$ matrix $\left(F_{I}\right)_{J K} \equiv \frac{\partial^{3} \mathcal{F}}{\partial a_{I} \partial a_{J} \partial a_{k}}$. 


\section{DF and Selberg matrix models}

The generic Virasoro conformal block can be considered as an analytic continuation in $N_{1}$ and $\mathrm{N}_{2}$ of the DF type integrals [16, 18]:

$$
\begin{gathered}
\mathcal{B}_{\Delta}\left(\Delta_{1}, \Delta_{2} ; \Delta_{3}, \Delta_{4} \mid q\right)= \\
\left\langle: e^{\alpha_{1} \phi(0)}:: e^{\alpha_{2} \phi(q)}:: e^{\alpha_{3} \phi(1)}:: e^{\alpha_{4} \phi(\infty)}:\left(\int_{0}^{q}: e^{b \phi(x)}: d x\right)^{N_{1}}\left(\int_{0}^{1}: e^{b \phi(y)}: d y\right)^{N_{2}}\right\rangle_{\text {free fields }} \sim \\
\sim q^{2 \alpha_{1} \alpha_{2}}(1-q)^{2 \alpha_{2} \alpha_{3}} \prod_{i=1}^{N_{1}} \int_{0}^{q} d x_{i} \prod_{k=1}^{N_{2}} \int_{0}^{1} d y_{k} \prod_{i<j}^{N_{1}}\left(x_{i}-x_{j}\right)^{2 b^{2}} \prod_{k<l}^{N_{2}}\left(y_{k}-y_{l}\right)^{2 b^{2}} \prod_{i, k}^{N_{1}}\left(x_{i}-y_{k}\right)^{2 b^{2}} . \\
\cdot \prod_{i} x_{i}^{2 \alpha_{1} b}\left(q-x_{i}\right)^{2 \alpha_{2} b}\left(1-x_{i}\right)^{2 \alpha_{3} b} \prod_{k} y_{k}^{2 \alpha_{1} b}\left(q-y_{k}\right)^{2 \alpha_{2} b}\left(1-y_{k}\right)^{2 \alpha_{3} b} \sim \\
\sim\left\langle\left\langle\exp \left\{2 \sum_{m=1}^{\infty} \frac{q^{m}}{m}\left(\alpha_{2}+b \sum_{i}^{N_{1}} x_{i}\right)\left(\alpha_{3}+b \sum_{k}^{N_{2}} y_{k}\right)\right\}\right\rangle_{N_{1}}\right\rangle_{N_{2}}
\end{gathered}
$$

Integrals of this kind are often called the $\beta$-ensemble, $\beta=b^{2}$ being the degree of the Van-derMonde determinant in the integrand.

The 1.h.s. is a free chiral field correlator, evaluated with the help of the Wick theorem, and the r.h.s. is a double average in two Selberg models,

$$
\begin{aligned}
\langle f(x)\rangle_{N_{1}} & \equiv S_{N_{1}}^{-1} \int_{0}^{1} f(x) \prod_{i<j}^{N_{1}}\left(x_{i}-x_{j}\right)^{2 b^{2}} \prod_{i=1}^{N_{1}} x_{i}^{2 \alpha_{1} b}\left(1-x_{i}\right)^{2 \alpha_{2} b} d x_{i}, \\
\langle g(y)\rangle_{N_{2}} & \equiv S_{N_{2}}^{-1} \int_{0}^{1} g(y) \prod_{k<l}^{N_{2}}\left(y_{k}-y_{l}\right)^{2 b^{2}} \prod_{k=1}^{N_{2}} y_{k}^{2 \alpha_{1} b}\left(1-y_{k}\right)^{2 \alpha_{3} b} d y_{k}
\end{aligned}
$$

These Selberg integrals are explicitly evaluated and are rational functions of $\alpha$ and $b$ parameters, decomposable into linear factors whenever $f$ and $g$ are the Jack polynomials $J^{\left(b^{2}\right)}$, see [20, 19] for details and references.

\section{A detailed scheme}

Now we are ready to draw a more detailed scheme as compared with Fig.1. With the notions introduced and defined above, the new picture looks like Fig.5. Below we briefly discuss the meaning of the three links with question marks in the low part of the table. If clarified, together with the already established Dotsenko-Fateev (DF) representation of conformal blocks these arrows would provide one possible proof of the AGT conjecture, the one based essentially on the matrix model technique. The structure of such a proof is outlined on another figure, Fig.6.

The proof of the AGT relation between the conformal block $B$ and the Nekrasov function $N$ is here actually substituted with a proof of a much simpler identity: between the genus zero resolvents associated with the $\beta$-deformed spectral curves of the (Dotsenko-Fateev)matrix and Seiberg-Witten models,

$$
\rho_{0 \mid 1}^{D F_{\beta}}=\rho_{0 \mid 1}^{S W_{\beta}}
$$

This relation (26) can be established by a direct check, which for $\beta=1$ has been performed in [14]. For $\beta \neq 1$ one still needs to know the $\beta$-deformed version of $\rho_{0 \mid 1}^{S W_{\beta}}$, which is provided 
rep.theory of

Virasoro algebra rep.theory

of $S_{\infty}$
Hurwitz

theory hypergeometric $\stackrel{c=\infty}{\longleftarrow}$ series

\begin{abstract}
conformal
\end{abstract}
blocks

$\stackrel{A G T}{=}$

Nekrasov

$\longleftarrow$

functions

LMNS

integrals

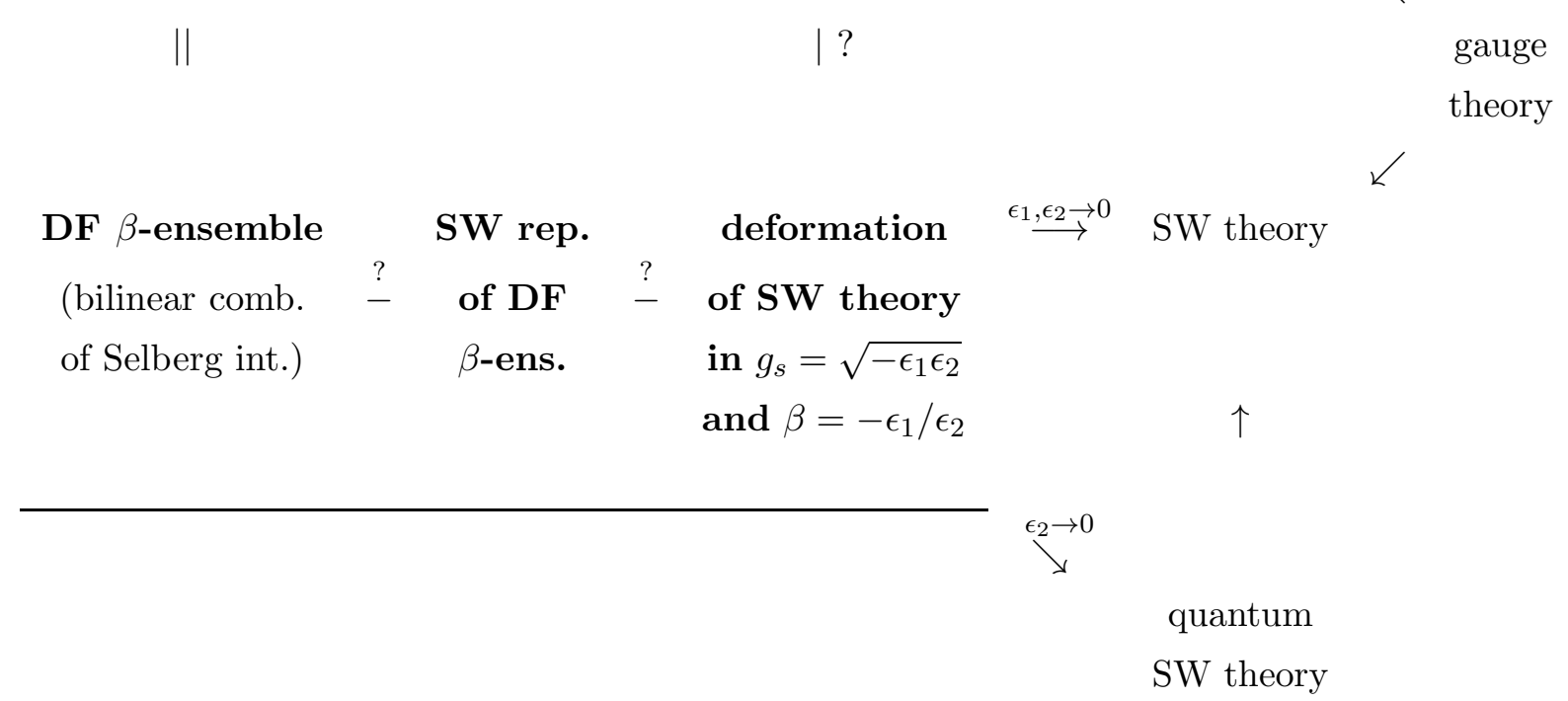

Figure 5: The detailed diagram of the AGT conjecture.

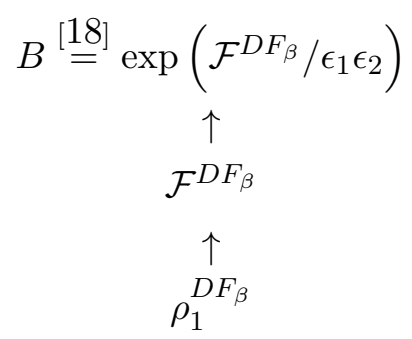

\section{SW procedure}

Top.Rec. $_{\beta}$

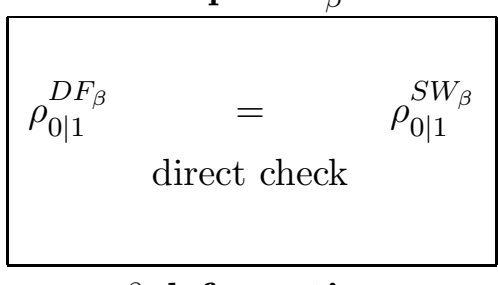

$\beta$-deformation

SW theory

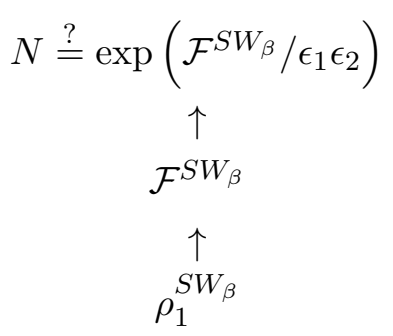

$\nearrow$

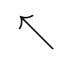

$\rho_{0 \mid 1}^{S W}$

$\uparrow$

gauge theory

Figure 6: The scheme of the proof of the AGT conjecture. 
by the generic procedure of $\beta$-deformation of the spectral curves, see s, 11 below. Once (26) is established, one lifts it by two canonical operations first to the equality of the full resolvents,

$$
\rho_{1}^{D F_{\beta}}=\rho_{1}^{S W_{\beta}}
$$

with the help of the ( $\beta$-deformed) topological recursion and, second, to equality of the free energies

$$
\mathcal{F}^{D F_{\beta}}=\mathcal{F}^{S W_{\beta}}
$$

with the help of the standard Seiberg-Witten procedure, which builds up the prepotential $\mathcal{F}$ from the Seiberg-Witten differential $\rho_{1}$. Finally, exponentiating the free energy provides the conformal blocks and the Nekrasov functions. In the case of conformal blocks, this equality,

$$
B \stackrel{18}{=} \exp \left(\mathcal{F}^{D F_{\beta}} / \epsilon_{1} \epsilon_{2}\right)
$$

is nothing but a new Dotsenko-Fateev type representation of conformal blocks with open integration contours introduced in [18. In the case of Nekrasov functions this statement,

$$
N \stackrel{?}{=} \exp \left(\mathcal{F}^{S W_{\beta}} / \epsilon_{1} \epsilon_{2}\right)
$$

remains to be proved, and the possibility of giving such a proof depends very much on the choice of a proper definition of Nekrasov functions: for various definitions see [35, 45].

In the remaining part of this paper we briefly comment on the main ingredients of this suggested proof, especially on the three canonical operations: $\beta$-deformation, topological recursion (i.e. $g_{s}$-deformation) and the SW procedure. All formulas below are given for the simplest 4-point spherical conformal block. Generalizations to arbitrary conformal blocks, at least, spherical and topic, and associated quiver gauge theories are straightforward.

\section{Topological recursion}

Switching on the string coupling $g_{s} \neq 0$, i.e. the deformation of the quasiclassical

$$
\mathcal{F}_{0}^{(m m)}=\mathcal{F}^{(m m)}\left(g_{s}=0\right)
$$

into the full $\mathcal{F}^{M A M O}$, has actually a functorial description, with no reference to matrix models. This lifting

$$
\left(\Sigma, \Omega_{0}\right) \longrightarrow \Omega=\sum_{p=0}^{\infty} g_{s}^{2 p} \Omega_{p}
$$

or, equivalently,

$$
\left(\Sigma, \Omega_{0}\right) \longrightarrow \tau \text {-function, }
$$

or even a "quantization" $\tau_{\text {quasicl }} \rightarrow \tau$, is now known under the name of topological recursion, where "topological" refers to the "genus expansion" in powers of $g_{s}$. It already has a numerous applications to different subjects, not explicitly related to matrix models.

The well-publicized part of the story, the AMM/EO construction [39]-42] consists of building up a hierarchy of poly-differentials (multi-resolvents) on the spectral curve $\Sigma$. It is a part of a more general theory, [41], covering also the decomposition formulas, i.e. the construction of integrability preserving intertwiners (of which the simplest example are $W$-representations of [46, 47]). This approach "implicitly" refers to the Virasoro constraints, i.e. the matrix model Ward identities [48], and is very general. However, it is not very practical if one needs to construct $\Omega$, because it involves an auxiliary construction of all unneeded, and far more complicated, multi-resolvents at intermediate steps. 
An alternative approach [49, 39, 50, 51, 52] builds

$$
\Omega=\sum_{p=0}^{\infty} g_{s}^{2 p} \Omega_{p}
$$

directly from $\Omega_{0}$, by writing and solving the difference-differential equation for $\Omega$, which involves recursion over $N \sim 1 / g_{s}$ (actually, over the matrix size), and follows from integrability properties of $\tau=e^{\mathcal{F}} / g_{s}^{2}$. It is explicitly known only in the case of Hermitean model, but there is nothing preventing one from developing a similar technique of the Harer-Zagier (HZ) recursion, for other matrix models.

Topological recursion: global description. Now we describe the topological recursion a bit more concretely. The construction implies that the whole set of multi-resolvents is intimately related to the $\widehat{U(1)}$ current $\hat{\mathcal{J}}(z)$ on the spectral curve $\Sigma$, with prescribed singularities: usually they are allowed at some fixed points (punctures) on $\Sigma$. In this approach the Virasoro constraints on partition function are written as

$$
\hat{\mathcal{P}}_{-}\left(\hat{\mathcal{J}}^{2}(z)\right) Z \equiv \hat{\mathcal{J}} * \hat{\mathcal{J}} Z=\oint_{C} \mathcal{K}\left(z, z^{\prime}\right)\left(\hat{\mathcal{J}}^{2}(z)\right) Z=0
$$

with a certain kernel $\mathcal{K}\left(z, z^{\prime}\right)$, made out of the free-field Green function on $\Sigma$. The current is also "shifted": $\hat{\mathcal{J}}(z) \longrightarrow \hat{\mathcal{J}}(z)+\Delta \hat{\mathcal{J}}(z)$ and partition function $Z$ depends on the choice of:

- the complex curve (Riemann surface) $\Sigma$,

- the Green function $\mathcal{K}\left(z, z^{\prime}\right)$, i.e. projection operator $\hat{\mathcal{P}}_{-}$,

- the punctures on $\Sigma$ and associated loop operator $\hat{\mathcal{J}}(z)$,

- the local coordinates in the vicinity of the punctures,

- the involution of the curve with punctures and loop operator,

- the shift $\Delta \mathcal{J}(z)$ on $\Sigma$,

- the contour $C$ which separates two sets of punctures.

If contour $C$ goes around an isolated puncture, $Z$ is actually defined by its infinitesimal vicinity and depends on behavior (the type of singularity) of $\hat{\mathcal{J}}(z)$ at this particular puncture. Coordinate dependence is reduced to the action of a unitary operator (Bogoliubov transform, and exponential of bilinear function of $\hat{\mathcal{J}}$ ) on $Z$.

If contour $C$ is moved away from the vicinity of the puncture, it can be decomposed into contours encircling all other punctures: this provides relations between $Z$ 's of different types, associated with different punctures, these are exactly the decomposition formulas.

Topological recursion: local description. The standard recursive loop equations [39]-[42] are reproduced from this global construction locally in the vicinity of a fixed point. To this end, one has to choose the local parameter $z$ in this vicinity and put

$$
\hat{\mathcal{J}}=V^{\prime}+g_{s}^{2} \hat{\nabla}
$$

where

$$
\hat{\nabla}(z)=\sum_{k=0}^{\infty} \zeta_{k}(z) \frac{\partial}{\partial T_{k}} \quad V^{\prime}(z)=\sum_{k=0}^{\infty} \tilde{T}_{k} v_{k}(z)
$$

and $v_{k}(z)$ and $\zeta_{k}(z)$ are the full sets of 1 -forms on $\Sigma$, related by the condition

$$
\hat{\nabla}(z) V^{\prime}\left(z^{\prime}\right)=B\left(z, z^{\prime}\right)
$$

where $B\left(z, z^{\prime}\right)$ is the Bergmann kernel, i.e. $(1,1)$ Green function $B\left(z, z^{\prime}\right)=<\partial \phi(z) \partial \phi\left(z^{\prime}\right)>$ on $\Sigma$. The integral in (31) gives rise to a multiplication map $\Omega_{\Sigma} \times \Omega_{\Sigma} \rightarrow \Omega_{\Sigma}$ on the space of 1-forms $\Omega_{\Sigma}$. For hyperelliptic curves, which are double coverings of the Riemann sphere, the contour 
$C$ is a finite set of contours encircling the ramification points and $\tilde{z}$ is the counterpart of $z$ on the other sheet. Then the kernel $K$ is actually a differential of the form $\frac{d z}{d \tilde{z}^{\prime}}$, which is a ratio of the $(1,0)$ Green function on $\Sigma$ (which is the primitive of the Bergmann kernel w.r.t. the second argument calculated from $z^{\prime}$ to $\left.\tilde{z}^{\prime}\right)$ and the Seiberg-Witten-Dijkraaf-Vafa differential 1 :

$$
K\left(z, z^{\prime}\right)=\frac{<\partial \phi(z) \phi\left(z^{\prime}\right)>-<\partial \phi(z) \phi\left(\tilde{z}^{\prime}\right)>}{\Omega_{D V}\left(z^{\prime}\right)-\Omega_{S W}\left(\tilde{z}^{\prime}\right)}
$$

See [41] and [53, 42] for details.

Substitution of (33) into (31) gives:

$$
\left(g_{s}^{2} \sum_{k, n \geq 0}\left(v_{k} * \zeta_{n}\right) \tilde{T}_{k} \frac{\partial}{\partial T_{n}}+\frac{g_{s}^{4}}{2} \sum_{k, l \geq 0}\left(\zeta_{k} * \zeta_{l}\right) \frac{\partial^{2}}{\partial T_{k} \partial T_{l}}+\frac{1}{2} \sum_{k, l \geq 0}\left(v_{k} * v_{l}\right) \tilde{T}_{k} \tilde{T}_{l}+\frac{1}{2} \operatorname{Tr}_{*} B\right) Z=0
$$

where the shift of the current $\Delta \mathcal{J}(z)$ is in charge of the $*$-trace of the Bergmann kernel. Expanding the products of 1-forms into linear combinations of $\zeta$ (no $v$ will arise due to projection property of the $*$-product), one obtains a one-dimensional set of constraints on $\log Z \equiv \sum_{p \geq 0} g_{s}^{2 p-2} \mathcal{F}^{(p)}$. They can be also written as recurrent relations for the multiresolvents

$$
\rho^{(p \mid m)}\left(z_{1}, \ldots, z_{m}\right)=\left.\hat{\nabla}\left(z_{1}\right) \ldots \hat{\nabla}\left(z_{m}\right) \mathcal{F}^{(p)}\right|_{T_{k}=\delta_{k, 1}}
$$

in the following form

$$
\begin{gathered}
\rho^{(p \mid m+1)}\left(z, z_{1}, \ldots, z_{m}\right)=\frac{1}{2} \operatorname{Tr}_{*} B(\bullet, \bullet) \delta_{p, 0} \delta_{m, 0}+\sum_{i=1}^{m} B\left(\bullet, z_{i}\right) * \rho^{(p \mid m)}\left(\bullet, z_{I / i}\right)+ \\
+\sum_{p_{1}=0}^{p} \sum_{J \subset I} \rho^{\left(p_{1} \mid m_{J}+1\right)}\left(\bullet, z_{J}\right) * \rho^{\left(p_{2} \mid m_{I / J}+1\right)}(\bullet, I / J)+\frac{1}{2} \operatorname{Tr}_{*} \rho^{(p-1 \mid m+2)}\left(\bullet, \bullet, z_{1}, \ldots, z_{m}\right)
\end{gathered}
$$

They are obtained simply by acting with operators $\hat{\nabla}$ on (31) and putting $T_{k}-\delta_{k, 1}=0$ afterwards. The terms with the Bergmann kernel come from the action of $\hat{\nabla}$ on $V^{\prime}$, action on the $V^{\prime} * V^{\prime}$ term gives rise to the trace of the Bergmann kernel. The notation here is as follows: the bullets, $\bullet$ mark arguments on which the $*$-product acts, two points are converted into a single $z$. If both bullets are arguments in the same function, we call the corresponding product $*$-trace, $T r_{*}$ : for, say, $H\left(z_{1}, z_{2}\right)=\sum_{m, n} H_{m n} \zeta_{m}\left(z_{1}\right) \zeta_{n}\left(z_{2}\right)$ the $*$-trace is $\operatorname{Tr}_{*} H(\bullet, \bullet)=\sum_{m, n} H_{m n}\left(\zeta_{m} * \zeta_{n}\right)(z)$.

\section{$11 \beta$-deformation}

$\beta$-ensembles differ from eigenvalue matrix models [29] by substitution of the second power of the Van-der-Monde determinant $\Delta(M)$ in the measure on the space of eigenvalues by arbitrary power $2 \beta$. This leads to a straightforward, but rather sophisticated deformation of integrals involving only traces and determinants of the integration $\beta$-ensemble variables. The $\beta$-character calculus [54, 24] which can be used to describe this deformation, automatically provides also the tools to handle the eigenvalue models with one external field, like Kontsevich model [55], GKM [27] and BGWM [28]. Character calculus in [24] is not, however, developed enough to describe $\beta$-deformations of the Kontsevich and DV phases in these models as well as of their integrability properties. In the context of this paper it is sufficient to know the $\beta$-deformations

${ }^{1}$ In simplest case of the sphere, $\Sigma_{H}: y_{H}^{2}(z)=z^{2}-4 S$ corresponding to the Hermitean one-matrix model 41 ]

$$
K\left(z, z^{\prime}\right)=\frac{d z}{d z^{\prime}} \frac{1}{z-z^{\prime}}\left(\frac{1}{y_{H}(z)}-\frac{1}{y_{H}\left(z^{\prime}\right)}\right)
$$


of the spectral curve and Virasoro constraints and thus of the AMM/EO topological recursion. Remarkably, these are the simplest things to $\beta$-deform, and one does need to know anything about the $\beta$-character calculus. In particular, formula (25) describes the $\beta$-ensemble with $\beta=b^{2}$. This is actually a simple deformation: it affects the spectral curve $\Sigma$ only through rescaling of coefficients, and it slightly changes the AMM/EO topological recursion (in particular, for $\beta \neq 1$ the $g_{s}$-expansion also includes odd powers of $g_{s}$, (50): contributions from open and non-oriented surfaces, "halves of their closed surface doubles").

$\beta$-deformation of the SW data. The SW data (20) for the $\beta$-ensemble is trivially deformed

$$
\left\{\begin{array}{c}
a_{I}=-\oint_{A_{I}} \rho_{1} \\
\frac{\partial \mathcal{F}}{\partial a_{I}}=-\beta \oint_{B_{I}} \rho_{1}
\end{array}\right.
$$

The genus zero one-point resolvent for $\beta$-ensembles looks like (see s.13 for the details)

$$
-\beta \rho_{0 \mid 1}^{2}+W^{\prime} \rho_{0 \mid 1}=f(z) \equiv\left(W^{\prime} \rho\right)_{+} \quad \rho_{0 \mid 1}(z)=\frac{W^{\prime}(z)-\sqrt{W^{\prime}(z)^{2}-4 \beta f(z)}}{2 \beta} d z
$$

and the spectral curve is

$$
\left(\rho_{0 \mid 1}(z)-\frac{W^{\prime}(z)}{2 \beta}\right)^{2}=y^{2}(z)=\frac{W^{\prime}(z)^{2}-4 \beta f(z)}{4 \beta^{2}}
$$

For the generic SW theory one should first represent $\rho_{0 \mid 1}$ at $\beta=1$ in this form with some $W^{\prime}(z)$ and $f(z)$ and then introduce $\beta$. For example, for the $S U(2)$ pure gauge theory (23) (here we subtracted the potential part from the genus zero resolvent, see s.13)

$$
\rho_{0 \mid 1}^{S U(2)}=\frac{\sqrt{\Lambda^{2} \cos z-E}}{\sqrt{2}} d z=\sqrt{\left(\Lambda \cos \frac{z}{2}\right)^{2}-E^{\prime}} d z \quad \longrightarrow \quad \rho_{0 \mid 1}^{S U(2)_{\beta}}=\frac{1}{\beta} \sqrt{\left(\Lambda \cos \frac{z}{2}\right)^{2}-\beta E^{\prime}}
$$

$\beta$-deformation of the Virasoro algebra (=loop equations). The AMM/EO topological recursion is easily $\beta$-deformed. For example, the Virasoro operators for Hermitean model change from

$$
-\frac{\partial}{\partial t_{n+2}}+\sum_{k} k t_{k} \frac{\partial}{\partial t_{k+n}}+\sum_{a+b=n} \frac{\partial^{2}}{\partial t_{a} \partial t_{b}}
$$

to

$$
-\frac{\partial}{\partial t_{n+2}}+\sum_{k} k t_{k} \frac{\partial}{\partial t_{k+n}}+\beta \sum_{a+b=n} \frac{\partial^{2}}{\partial t_{a} \partial t_{b}}+(1-\beta)(n+1) \frac{\partial}{\partial t_{n}}
$$

More generally, one constructs the $\beta$-deformation of the topological recursion completely along the line of the previous section, with only two ingredients deformed. First of all, one has to deform the spectral curve (and, certainly, the related quantities like $\mathcal{K}\left(z, z^{\prime}\right)$ and the Bergmann kernel) and, then, the Sugawara construction, which is behind (31). The latter deformation is well-known and can be read off immediately using the standard conformal matrix model technique [56, 13]: one has to replace (31) with

$$
\hat{\mathcal{P}}_{-}\left(\hat{\mathcal{J}}^{2}(z)+\left(b-\frac{1}{b}\right) \partial_{z} \hat{\mathcal{J}}\right) Z=0
$$

It is yet unknown how does the $\beta$-deformation affects the integrability-inspired HZ topological recursion, but one can also assume that the changes will not be too drastic. 


\section{The LMNS double deformation of SW theory}

As clear from consideration of the previous sections, one can write the full free energy of the $\beta$-ensemble as

$$
\mathcal{F}^{(m m), \beta}=\text { top.rec. } \beta\left[\mathcal{F}_{0}^{(m m), \beta}\right]
$$

where the r.h.s. involves only functorial operation of topological recursion and the quasiclassical (genus zero) free energy. This means that the r.h.s. is fully defined by nothing but the SW pair $\left(\Sigma, \Omega_{0}\right)$.

Moreover, the same operation can be applied to the arbitrary SW pair $\left(\Sigma, \Omega_{0}^{S W}\right)$ to obtain a $\beta, g_{s}$ double deformation

$$
\mathcal{F}^{S W, \beta}=\text { top.rec. } \beta\left[\mathcal{F}_{0}^{S W, \beta}\right]
$$

The Nekrasov functions have several different definitions, see [35, 45] for some of them. One of the definitions implies that they arise as a double deformation of the (exponentiated) gauge theory SW prepotential by two parameters $\epsilon_{1}, \epsilon_{2}$. This double deformation can presumably be alternatively described as switching on $\beta \neq 1$ and $g_{s} \neq 0$ with the usual AGT identifications (19), $\beta=b^{2}$. In other words, one can define the Nekrasov functions as

$$
\log Z_{N e k}^{S U(2)}=\mathcal{F}^{S U(2), \beta}=\text { top.rec. } \beta\left[\mathcal{F}_{0}^{S U(2), \beta}\right]
$$

where at the r.h.s. stands just the usual gauge theory SW prepotential.

\section{Identification of $\mathrm{SW}$ and $\beta$-ensemble differentials $\Omega_{0}$ and spectral curves}

Now in order to establish the AGT conjecture one has to check first that the SW data coincides in the both cases: in the SW theory and in the $\beta$-ensemble. Then, applying the topological recursion, one lifts the both to the full free energies which then also coincide.

SW data in $\beta$-ensembles. Assume one starts from the $\beta$-ensemble

$$
Z \sim \int \prod_{i} d z_{i} \exp \left(-\frac{1}{g_{s}} W\left(z_{i}\right)\right) \prod_{i<j}\left(z_{i}-z_{j}\right)^{2 \beta}
$$

Then, using the invariance under the shift of integration variables $\delta z_{i} \sim z_{i}^{n+1}$ with $n \geq-1$, one immediately obtains (the double brackets here mean integration with the $\beta$-ensemble measure (48) )

$$
\left\langle 2 \beta g_{s} \sum_{i<j} \frac{z_{i}^{n+1}-z_{j}^{n+1}}{z_{i}-z_{j}}-\sum_{i} W^{\prime}\left(z_{i}\right) z_{i}^{n+1}+g_{s} \sum_{i} n z_{i}^{n}\right\rangle=0
$$

Summing up these identities over $n$ with the weights $\xi^{-n-2}$, one rewrites these Virasoro constraints in the form of a loop equation for the one-point resolvents:

$$
\beta \rho_{1}^{2}(\xi)-W^{\prime}(\xi) \rho_{1}(\xi)+f(\xi)=(1-\beta) g_{s} \frac{\partial \rho_{1}(\xi)}{\partial \xi}-\beta g_{s}^{2} \rho_{2}(\xi, \xi)
$$

where

$$
\rho_{k}\left(\xi_{1}, \ldots, \xi_{k}\right) \equiv g_{s}^{k}\left\langle\left\langle\prod_{a=1}^{k} \sum_{i} \frac{1}{\xi_{a}-z_{i}}\right\rangle_{c}, \quad f(\xi) \equiv\left\langle\left\langle\sum_{i} \frac{W^{\prime}(\xi)-W^{\prime}\left(z_{i}\right)}{\xi-z_{i}}\right\rangle\right.\right.
$$


and the subscript $c$ means the connected correlator. Solving the loop equation (50) in the leading order in the string coupling constant $g_{s}$, one obtains (compare with (40), the sign is chosen here in order to guarantee the correct behaviour at large $\xi$ )

$$
\rho_{0 \mid 1}(\xi)=\frac{W^{\prime}(\xi)-\sqrt{W^{\prime 2}(\xi)-4 \beta f(\xi)}}{2 \beta}
$$

In fact, it is usually more convenient to define $\rho_{0 \mid 1}(z)$ with the potential part subtracted, not changing the definition of higher order resolvents, and from now on we use the one-point resolvent defined exactly in such a way. The spectral curve and the SW differential are then

$$
\begin{gathered}
\Omega_{0}=y d z \\
\Sigma: \quad y^{2}=\frac{W^{\prime 2}(\xi)-4 \beta f(\xi)}{4 \beta^{2}}
\end{gathered}
$$

DF integrals as $\beta$-ensembles. Choosing now the potential

$$
W(\xi)=-2 \epsilon_{1} \sum_{k=1}^{3} \alpha_{k} \log \left(\xi-q_{k}\right), \quad\left\{q_{1}, q_{2}, q_{3}\right\}=\{0, q, 1\}
$$

one reproduces the $\beta$-ensemble (25) and obtains (after rescaling $y$ ) from (53) the following SW data (see [14, (3.10)], [22, (3.38)], [16, (46)]):

$$
\begin{gathered}
\Omega_{0}=y d z \\
\Sigma: \quad y^{2}=\frac{M_{-}^{2}}{z^{2}}+\frac{M_{+}^{2}}{(z-1)^{2}}+\frac{m_{+}^{2}}{(z-q)^{2}}-\frac{M_{+}^{2}+M_{-}^{2}+m_{+}^{2}-m_{-}^{2}}{z(z-1)}-\frac{(1-q) u}{z(z-1)(z-q)}
\end{gathered}
$$

Here

$$
m_{ \pm}=\alpha_{2,4}=\frac{\mu_{1} \pm \mu_{2}}{2}, \quad M_{ \pm}=\alpha_{3,1}=\frac{\mu_{3} \pm \mu_{4}}{2}
$$

Note that the same spectral curve can be obtained via absolutely different procedure of studying the differential equation satisfied by the conformal block with a degenerate field inserted [23, (2.24)], [25, (88)].

SW theory vs. planar limit of the DF $\beta$-ensemble. In order to compare the SW data (55) with that of the corresponding SW theory, eq.(21), one has to make the change of variables in this latter

$$
e^{i \phi}=z, \quad\left(p-\frac{\left(\mu_{1}+\mu_{2}-2 h\right) e^{2 i \phi}+q\left(\mu_{3}+\mu_{4}-2 h\right)}{2\left(e^{i \phi}-1\right)\left(e^{i \phi}-q\right)}\right) e^{-i \phi}=y
$$

which, indeed, leads to the SW pair (55) upon the identification

$$
u=\frac{1+q}{1-q} E-\frac{\left(M_{+}-m_{+}\right)^{2}}{1-q^{2}}+\frac{q \mu_{1} \mu_{2}+\mu_{3} \mu_{4}}{1-q}
$$

Thus, we prove that the SW data for the DF $\beta$-ensemble and the $S U(2)$ gauge theory with four fundamental matter hypermultiplets coincide. Then they would keep to coincide after the topological recursion applied, i.e. the conformal block is equal to the Nekrasov functions (defined by the topological recursion). 


\section{The pure gauge limit}

The most familiar is the gauge SW theory in the "pure gauge" case, when the four fundamental masses are taken to infinity, while $q \rightarrow 0$, so that $\Lambda^{4}=q m_{1} m_{2} m_{3} m_{4}$ remains finite. Then the SW pair, the spectral curve and the differential become (23). In order to see this one suffices to make the change of variables in (23)

$$
e^{i \phi}=z, \quad p e^{-i \phi}=y
$$

and immediately to come to

$$
\begin{aligned}
\Omega_{0}^{S U(2), p g} & =y d z \\
\Sigma^{(S U(2), p g}: \quad y^{2} & =\frac{\Lambda^{2}}{z}+\frac{E}{z^{2}}+\frac{\Lambda^{2}}{z^{3}}
\end{aligned}
$$

This spectral curve is exactly the curve which describes the pure gauge theory limit on the conformal side.

Note that the DF $\beta$-ensemble in the pure gauge limit looks rather different from (25)). This limit is obtained by presenting the $\beta$-ensemble integral (25) as an average of the product of two Selberg integrals and further taking the limit of these Selberg integrals. According to [24] the final answer is given by

$$
\begin{gathered}
B_{*}(\Delta \mid \Lambda)=\lim _{\substack{\Delta_{1}, \Delta_{2}, \Delta_{3}, \Delta_{4} \rightarrow \infty, q \rightarrow 0 \\
q\left(\Delta_{2}-\Delta_{1}\right)\left(\Delta_{3}-\Delta_{4}\right) \equiv \Lambda^{4}}}\left[B\left(\Delta_{1}, \Delta_{2}, \Delta_{3}, \Delta_{4} ; \Delta, c \mid q\right)\right]= \\
=\left.\exp \left(-2 \beta \sum_{k=1}^{\infty} \frac{\Lambda^{4 k}}{k} \frac{\partial^{2}}{\partial t_{k}^{+} \partial t_{k}^{-}}\right) Z_{*}\left(n_{+} \mid t^{+}\right) Z_{*}\left(n_{-} \mid t^{-}\right)\right|_{t=0}
\end{gathered}
$$

where $Z_{*}(n \mid t)$ is the partition function of the Brezin-Gross-Witten model in the character phase,

$$
Z_{*}\left(n \mid t_{k}=\operatorname{tr} \Psi^{k} / k\right)=\frac{1}{\operatorname{Vol}_{\beta}(n)} \int_{n \times n}[d U]_{\beta} e^{\operatorname{tr} U^{+}+\operatorname{tr} \Psi U}
$$

and

$$
n_{ \pm}= \pm \frac{2 a}{\epsilon_{1}}, \quad a=\alpha-\frac{\epsilon_{1}+\epsilon_{2}}{2}
$$

\section{Conclusion}

In this paper we presented a possible strategy for proving the AGT relation between conformal blocks and Nekrasov functions, based on existence of the canonical double deformation of arbitrary Seiberg-Witten prepotentials with the help of the topological recursion. This recursion can be applied both to the gauge theory SW prepotentials and to the Dotsenko-Fateev free energies. Since the spectral curves and SW differentials in these two cases are known to coincide, this actually implies the AGT relation.

To make this argument into a constructive identification, with all steps explicit, a better understanding is desirable of the $\mathrm{HZ}$ version of the topological recursion, and thus of integrability properties of non-Gaussian and $\beta$-deformed matrix models.

\section{Acknowledgements}

Our work is partly supported by Ministry of Education and Science of the Russian Federation under contract 02.740.11.0608, by RFBR grants 10-02-00509-a (A.Mir.), and 10-02-00499 (A.Mor. \& Sh.Sh.), by joint grants 09-02-90493-Ukr, 09-01-92440-CE, 09-02-91005-ANF, 10-0292109-Yaf-a. The work of A.Morozov was also supported in part by CNRS. 


\section{Appendix I. On HZ topological recursion}

The celebrated Hermitian model resolvent [39] (to make a contact with the standard matrix model notations, in this Appendix we use the definition of the resolvent without subtracting the potential term and rescale $y$ by a factor of 2)

$$
\rho_{N}^{H M}(x)=\frac{x-y}{2}+\frac{N}{y^{5}}+\frac{21 N\left(x^{2}+N\right)}{y^{11}}+\frac{11 N\left(135 z^{4}+558 N z^{2}+158 N^{2}\right)}{y^{17}(z)}+\ldots
$$

with $y^{2}=x^{2}-4 N$ actually satisfies the difference equation [39]

$$
\rho_{N+1}-2 \rho_{N}+\rho_{N-1}=\rho_{N}^{\prime \prime} / N
$$

which can be also rewritten as the differential equation [50, 52 ]

$$
-\rho_{N}^{\prime \prime \prime}+y^{2} \rho_{N}^{\prime}-x \rho_{N}+2 N=0
$$

Equation (65) (or, equivalently, (66)) is very different from the usual topological recursion. Still, it completely reproduces the asymptotic series (64). As explained in [39, 50] such equations follow from integrability of the model, in this particular case from the lowest Toda-chain equation [26]

$$
\frac{Z_{N+1}^{H M} Z_{N-1}^{H M}}{Z_{N}^{H M}}=\frac{\partial^{2}}{\partial t_{1}^{2}} \log Z_{N}^{H M}
$$

where

$$
Z_{N}^{H M}=\frac{\int_{n \times n} d M e^{-\operatorname{tr} M^{2} / 2+\sum_{k} t_{k} \operatorname{tr} M^{k}}}{\int_{n \times n} d M e^{-\operatorname{tr} M^{2} / 2}}
$$

is the partition function of the Gaussian Hermitian matrix model. As is also explained in [50] (and even earlier in [39]) it is often very convenient to consider not the matrix model at particular value of $N$, but the generating function w.r.t. $N$, the "grand-ensemble" partition function

$$
Z^{H M}(\lambda)=\sum_{N=0}^{\infty} \lambda^{N} Z_{N}^{H M}
$$

and its derivatives, such as the "grand-ensemble" resolvent:

$$
\hat{\rho}(x \mid \lambda)=\sum_{N=0}^{\infty} \lambda^{N} \rho(x \mid N)
$$

This function is in many respects more clever than its finite $N$ counterpart: just like $\rho_{N}$ it is given by an asymptotic series (see eq.(42) in [51]), but this time the series can be explicitly summed into an error function [39]:

$$
\hat{\rho}^{H M}(x \mid \lambda)=\sum_{k=0}^{\infty} \frac{\lambda(1+\lambda)^{k}}{(1-\lambda)^{k+2}} \frac{(2 k-1) ! !}{x^{2 k+1}}=\frac{i \lambda}{(1-\lambda) \sqrt{1-\lambda^{2}}} \operatorname{erf}\left(i z \frac{1-\lambda}{1+\lambda}\right)
$$

The function $\hat{\rho}$ satisfies

$$
\lambda \partial_{\lambda}\left(\frac{(1-\lambda)^{2}}{\lambda} \hat{\rho}\right)=\hat{\rho}^{\prime \prime}
$$

which is obviously equivalent to (65), or

$$
-\hat{\rho}^{\prime \prime \prime}+\left(x^{2}-4 \lambda \partial_{\lambda}\right) \hat{\rho}^{\prime}-x \hat{\rho}+\frac{2 \lambda}{(1-\lambda)^{2}}=0
$$

which is obviously equivalent to (666). See also [49, 52, 39, 51] for more details. Integrability inspired equations of this type are often useful in various applications of matrix models (and, perhaps, will be useful in applications to the AGT conjecture). To illustrate their usefulness, in the next Appendix we use eq. (65) to prove the Seiberg-Witten representation of the Gaussian Hermitian model free energy exactly (in all genera). 


\section{Appendix II. Seiberg-Witten representation of the Gaussian- model partition function}

The SW representation (20) of the matrix model free energy is not known widely enough, despite its extreme conceptual importance. It is only briefly mentioned even in recent matrix model reviews, see, for example, [57, 53, 40. Moreover, its general prove is not actually available at the moment.

In this Appendix we demonstrate how this representation works in the simplest case of the Gaussian Hermitian model (with $\beta=1$ ). In this case

$$
Z_{N}=\frac{1}{V_{N}} \int_{N \times N} d M e^{-\frac{1}{g} \operatorname{tr} M^{2}}=\frac{g^{N^{2} / 2}}{V_{N}} \int_{N \times N} d M e^{-\operatorname{tr} M^{2}}=g^{N^{2} / 2} \prod_{k=1}^{N-1} k !
$$

where $V_{N}$ is the volume of the unitary group. Thus

$$
F_{N}=\log Z_{N}=\frac{N^{2}}{2} \log g+\sum_{k=1}^{N-1} \log (k !)
$$

This free energy actually gets contributions from all genera, since in genus expansion one uses the variables $S=g N$ and $g$. Equivalently, one can keep $g=1$ and consider the genus expansion simply as an asymptotical expansion at large $N$. Convenient for this purpose is the EulerMcLaurin summation formula [58]

$$
\sum_{k=0}^{N-1} f(k)=\int_{0}^{N} f(x) d x+\frac{B_{1}}{1 !}(f(N)-f(0))+\sum_{k=2} \frac{B_{k}}{k !}\left[f^{(k-1)}(N)-f^{(k-1)}(0)\right]
$$

i.e.

$$
\frac{\partial}{\partial N} \sum_{k=1}^{N-1} f(k)=f(N)+\frac{B_{1}}{1 !} f^{\prime}(N)+\frac{B_{2}}{2 !} f^{\prime \prime}(N)+\frac{B_{4}}{4 !} f^{\prime \prime \prime \prime}(N)+\ldots
$$

where $B_{k}$ are the Bernoulli numbers [59], $\sum_{k=0} B_{k} x^{k} / k !=x /\left(e^{x}-1\right), B_{0}=1, B_{1}=-1 / 2, B_{2}=$ $1 / 6, B_{3}=0, B_{4}=-1 / 30, \ldots$ Applying (77) with

$$
f(z)=\Gamma(z+1)=(z+1 / 2) \log z-z+\log \sqrt{2 \pi}+\sum_{m=1}^{\infty} \frac{B_{2 m}}{2 m(2 m-1) z^{2 m-1}}
$$

one finds

$$
\begin{gathered}
\frac{\partial F_{N}}{\partial N}=(N \log (g N)-N)+\log \sqrt{2 \pi}-\frac{1}{12 N}+\frac{1}{120 N^{3}}-\frac{1}{252 N^{5}}+\ldots= \\
=(N \log (g N)-N)+\log \sqrt{2 \pi}-\sum_{g=1}^{\infty} \frac{B_{2 g}}{2 g} \frac{1}{N^{2 g-1}}
\end{gathered}
$$

Note that only the odd powers of $1 / N$ survive in the series at the r.h.s. To obtain (79) one makes

use of the bilinear identity between the Bernoulli numbers, $\sum_{i+j=k} \frac{(2 k-2) !}{(2 i) !(2 j) !} B_{2 i} B_{2 j}=-\frac{1}{2 k} B_{2 k}$, $k>1$.

The aim of this appendix is to reproduce this expansion from the one-point resolvent (64)

$$
\rho_{1}(z \mid N) \stackrel{39}{=}-\frac{y(z)}{2}+\frac{N}{y^{5}(z)}+\frac{21 N\left(z^{2}+N\right)}{y^{11}(z)}+\frac{11 N\left(135 z^{4}+558 N z^{2}+158 N^{2}\right)}{y^{17}(z)}+\ldots
$$

where $y^{2}(z)=z^{4}-4 N$. The $A$-period of $\rho_{1}$ is simple:

$$
a=-\oint_{A} \rho_{1}(z \mid N) d z=-\oint_{-\sqrt{4 N}}^{\sqrt{4 N}} \rho_{1}(z \mid N) d z=N
$$


(hereafter, we use a peculiar notation $\oint_{a}^{b}$ for the integral along the contour which goes around the points $a$ and $b$ ). Only the first (genus-zero) term in (80) contributes to the $A$-period, because the integration contour can be taken to infinity and $\rho^{(1 \mid p)} \sim z^{-4 p-1}$ as $z \rightarrow \infty$. The $B$-period is more complicated:

$$
\frac{\partial F}{\partial a}=-\oint_{B} \rho_{1}(z \mid N) d z=-\oint_{\sqrt{4 N}}^{\infty} \rho_{1}(z \mid N) d z
$$

It can even seem that the contributions of higher genera diverge, since, for example, the integral $\int_{-\sqrt{4 N}}^{\infty} \frac{d z}{\left(z^{2}-4 N\right)^{k / 2}}$ along the ray $(-\sqrt{4 N}, \infty)$ diverges when $k \geq 0$. This is, however, not the case: integrals of $\rho_{1}$ are actually finite for $k \geq 1$, because the integration is along the contour surrounding the points $\sqrt{4 N}$ and $\infty$, not along a segment or a ray, thus, the contour can be taken away from the singularities. This works exactly in the same way as in the case of the integral $\int_{-\sqrt{4 N}}^{\sqrt{4 N}} \frac{d z}{\left(z^{2}-4 N\right)^{k / 2}}$, the same phenomenon also ensures the finiteness of the corrected Bohr-Sommerfeld periods in [12].

Actually, making the change of variables $\frac{z}{\sqrt{4 N}}=\frac{2-\zeta}{\zeta}$, one reduces the integral to the $B$ function,

$$
\frac{1}{2} \oint_{0}^{1} \zeta^{a-1}(1-\zeta)^{b-1} d \zeta=\frac{\Gamma(a) \Gamma(b)}{\Gamma(a+b)}
$$

in the following way:

$$
\oint_{\sqrt{4 N}}^{\infty} \frac{d z}{\left(z^{2}-4 N\right)^{p / 2}}=\frac{2^{p+1}}{(4 N)^{p / 2}} \oint_{0}^{1} \zeta^{p-2}(1-\zeta)^{-p / 2} d \zeta=\frac{2^{p+1}}{(4 N)^{p / 2}} \frac{\Gamma(p-1) \Gamma(1-p / 2)}{\Gamma(p / 2)}
$$

To see why the integral does not diverge, say for $a \geq 0$, one can deform the contour into a dumbbell, when the circle around $\zeta=0$ has a small radius $r$. Then the contribution from the segment diverges as $\frac{r^{a}}{2 a}\left(1-e^{2 \pi i a}\right)$, while the integral along the left circle is $\frac{r^{a}}{2} \oint_{0}^{2 \pi} e^{i a \phi} d \phi=$ $\frac{r^{a}}{2 i a}\left(e^{2 \pi i a}-1\right)$. Clearly, these two leading divergencies cancel each other.

With this prescription for calculation of $B$-period integrals, one finds

$$
\begin{gathered}
-\oint_{\sqrt{4 N}}^{\infty} \frac{y(z)}{2} d z=-\lim _{\Lambda \rightarrow \infty} \oint_{\sqrt{4 N}}^{\Lambda} \frac{y(z)}{2} d z=-\frac{\Lambda^{2}}{2}-(N \log N-N)+2 N \log 2 \Lambda+O\left(\frac{N}{\Lambda^{2}}\right) \\
\oint_{\sqrt{4 N}}^{\infty} \frac{N d z}{y^{5}(z)}=\oint_{0}^{1} \frac{\zeta^{3} d \zeta}{128 N(1-\zeta)^{5 / 2}}=\frac{1}{12 N} \\
\oint_{\sqrt{4 N}}^{\infty} \frac{21 N\left(z^{2}+N\right)}{y^{11}(z)} d z=\oint_{0}^{1} \frac{21 \zeta^{7}\left(16-16 \zeta+5 \zeta^{2}\right)}{524288 N^{3}(1-\zeta)^{11 / 2}} d \zeta=-\frac{1}{120 N^{3}} \\
\oint_{\sqrt{4 N}}^{\infty} \frac{11 N\left(135 z^{4}+558 N z^{2}+158 N^{2}\right)}{y^{17}(z)} d z=\oint_{0}^{1} \frac{11 \zeta^{11}\left(17280-34560 \zeta+30384 \zeta^{2}-13104 \zeta^{3}+2275 \zeta^{4}\right)}{1073741824 N^{5}(1-\zeta)^{17 / 2}} d \zeta=\frac{1}{252 N^{5}}
\end{gathered}
$$

so that the first terms of the expansion (79) are reproduced. Clearly, there is an identity

$$
-\oint_{B} \rho_{1}(z \mid N) d z=\frac{\partial F_{N}}{\partial a}+\text { const }_{1}+\text { const }_{2} \cdot N
$$

i.e. the derivative of the free energy is reproduced modulo $N$-constant and $N$-linear terms (they can be removed into redefinition/rescaling of various quantities such as $g, \Lambda$ and $Z$ itself).

The simplest way to check this identity in the generic form is to use equation (65):

$$
\rho_{1}(z \mid N+1)-2 \rho_{1}(z \mid N)+\rho_{1}(z \mid N-1)=\frac{1}{N} \frac{\partial^{2}}{\partial z^{2}} \rho_{1}(z \mid N)
$$

This equation directly implies that

$$
\oint_{B} \rho_{1}(z \mid N+1) d z-2 \oint_{B} \rho_{1}(z \mid N) d z+\oint_{B} \rho_{1}(z \mid N-1) d z=\frac{1}{N} \oint_{B} \frac{\partial^{2}}{\partial z^{2}} \rho_{1}(z \mid N) d z
$$


The $B$-contour integral of the full derivative does not vanish, as one could naively expect. The non-vanishing contribution comes from the genus zero part of the function:

$$
\frac{1}{N} \oint_{B} \frac{\partial^{2}}{\partial z^{2}} \rho_{1}(z \mid N)=-\frac{1}{N} \oint_{B} \frac{\partial^{2}}{\partial z^{2}}\left(\frac{y(z)}{2}\right) d z=-\frac{1}{N}
$$

All higher genera contributions vanish. Therefore, we proves that

$$
\oint_{B} \rho_{1}(z \mid N+1) d z-2 \oint_{B} \rho_{1}(z \mid N) d z+\oint_{B} \rho_{1}(z \mid N-1) d z=-\frac{1}{N}
$$

Precisely the same equation is satisfied by the r.h.s. of (89):

$$
F_{N}=\sum_{k=1}^{N-1} \log k ! \quad \Longrightarrow \quad F_{N+1}-2 F_{N}+F_{N-1}=\log N !-\log (N-1) !=\log N
$$

and

$$
\frac{\partial}{\partial a}\left(F_{N+1}-2 F_{N}+F_{N-1}\right)=\frac{1}{N}
$$

Therefore, the l.h.s. and r.h.s. of (89) satisfy one and the same equation. This equation has any linear function of $N$ as a kernel. This completes the proof.

\section{Appendix III. Limiting cases of AGT relation}

Conformal blocks and Nekrasov partition functions, which are identified by the AGT relation, depend on numerous free parameters (external and internal dimensions, $\epsilon$ 's), and one can look at various limits at the boundary of these moduli spaces. These limits are often highly non-

\begin{tabular}{|c|c|c|c|c|}
\hline limit & AGT & CFT & SW theory & $\beta$-ensemble \\
\hline interior of moduli space & [1] & 33 & 60, 37] & DF $\beta$-ensemble \\
\hline $\begin{array}{c}\Delta_{i, e x t} \rightarrow \infty \\
i=1 \ldots \kappa\end{array}$ & 4 & & $60,37,38$ & $\begin{array}{c}\text { DF } \beta \text {-ensemble if } \kappa \leq N_{C} \\
\text { Unitary } \beta \text {-ensembles otherwise }\end{array}$ \\
\hline$\Delta_{i n t} \rightarrow \infty$ & 9, 11 & 61 & perturbative regime & $?$ \\
\hline $\begin{array}{c}c \rightarrow \infty \\
\left(g_{s}=\sqrt{\epsilon_{1} \epsilon_{2}} \rightarrow 0\right)\end{array}$ & 10 & & & $?$ \\
\hline$\epsilon_{2} \rightarrow 0$ & 7., 12 & & quantized SW theory & $?$ \\
\hline$\Delta \rightarrow$ degenerate value & {$[6,5$} & [33, 62] & & [15] \\
\hline
\end{tabular}
trivial. When translated into the language of matrix models they can be also used to interrelate different $\beta$-ensembles, very much in the spirit of [41. In this Appendix we briefly review what is currently known about these limiting cases, see the following Table.

In the very left column of the Table only the quantities that becomes zero or infinite are written. For instance, the record $\Delta_{\text {ext }} \rightarrow \infty$ in the second row implies that all the external dimensions and the central charge remain finite, while the record $c \rightarrow \infty$ in the fourth row means that all the conformal dimensions are kept finite.

Originally the AGT conjecture relates conformally invariant theories in $2 d$ and in $4 d 2$ This means that one deals with the $\mathcal{N}=2$ SYM theory with additional adjoint, bifundamental or fundamental hypermultiplets, adjusted to guarantee vanishing of the $\beta$-function (e.g. $N_{A}=1$ or $N_{F}=2 N_{C}$ for the simple $S U\left(N_{C}\right)$ theories).

\footnotetext{
${ }^{2}$ Restriction to $4 d$ is actually inessential: dimension can be also 3,5 or 6 .
} 
The conformal invariance is broken through the dimensional transmutation when masses of some hypermultiplets are taken to infinity.

Another aspect of the AGT conjecture is that it occurs for the $\epsilon$-deformed SYM theories, where the $4 d$ Lorentz invariance is violated by peculiar graviphoton backgrounds. The limit of $g_{s}=\sqrt{-\epsilon_{1} \epsilon_{2}} \rightarrow 0$, leading to the ordinary SW theory, is singular. Remarkably, it is not always a naive planar limit in the matrix-model language: of interest are rather peculiar double-scaling limits, when the DF $\beta$-ensemble reduces to another, still non-trivial $\beta$-ensemble, not just to a quasiclassical approximation.

In particular, in the pure gauge limit what occurs are unitary $\beta$-ensembles [24], which are very interesting and deserve further investigation.

A special attention is recently attracted to the double-scaling limit $\epsilon_{2} \rightarrow 0$ [7]. It is interesting, because in this limit a non-trivial dependence on the single $\epsilon$-parameter, $\epsilon_{1}$, survives, despite $g_{s} \rightarrow 0$, and the SW theory is quantized in the most naive way: by switching from the classical integrable system [31] to its direct quantum counterpart, and from the spectral curves to the associated Baxter equation. The SW representation (20) for the free energy now involves exact (quantum) Bohr-Sommerfeld periods [12]: the monodromies of the wave functions of (the Fourier transform of) the Baxter equation, which are simultaneously non-perturbatively corrected Harish-Chandra functions [25. Moreover, the wave function itself has a wonderful interpretation in terms of CFT: it is equal to the conformal block with additional insertion of the degenerate primary [63, 17, 21, 23, 25], associated with a surface operator insertion [8] in the M5/SYM language. Remarkably, such insertions into the conformal block can be also made for $\epsilon_{2} \neq 0$, which corresponds to switching on Whitham times, 25], still, their exact role in the AGT relation has to be further clarified. Also an adequate matrix model description of the Baxter wave function is an open problem.

\section{References}

[1] L.Alday, D.Gaiotto and Y.Tachikawa, Lett.Math.Phys. 91 (2010) 167-197, arXiv:0906.3219

[2] N.Wyllard, JHEP 0911 (2009) 002, arXiv:0907.2189;

N.Drukker, D.Morrison and T.Okuda, JHEP 0909 (2009) 031, arXiv:0907.2593;

Andrey Mironov, Sergey Mironov, Alexei Morozov and Andrey Morozov, arXiv:0908.2064;

S.Iguri and C.Nunez, JHEP 11 (2009) 090 , arXiv:0908.3460;

D.Nanopoulos and D.Xie, arXiv:0908.4409; JHEP 1003 (2010) 043, arXiv:0911.1990; arXiv:1005.1350; arXiv:1006.3486;

N.Drukker, J.Gomis, T.Okuda and J.Teschner, JHEP 1002 (2010) 057, arXiv:0909.1105;

R.Dijkgraaf and C.Vafa, arXiv:0909.2453;

A.Gadde, E.Pomoni, L.Rastelli and S.Razamat, JHEP 1003 (2010) 032, arXiv:0910.2225;

L.Alday, F.Benini and Y.Tachikawa, Phys.Rev.Lett. 105 (2010) 141601, arXiv:0909.4776;

H.Awata and Y.Yamada, JHEP 1001 (2010) 125, arXiv:0910.4431; arXiv:1004.5122;

S.Kanno, Y.Matsuo, S.Shiba and Y.Tachikawa, Phys.Rev. D81 (2010) 046004, arXiv:0911.4787;

G.Bonelli and A.Tanzini, arXiv:0909.4031;

J.-F.Wu and Y.Zhou, arXiv:0911.1922;

L.Hadasz, Z.Jaskolski and P.Suchanek, arXiv:0911.2353, arXiv:1004.1841,

G.Giribet, JHEP 01 (2010) 097, arXiv:0912.1930;

V.Alba and And.Morozov, Nucl.Phys. B840 (2010) 441-468, arXiv:0912.2535;

M.Fujita, Y.Hatsuda, Y.Koyama and T.-Sh.Tai, JHEP 1003 (2010) 046, arXiv:0912.2988;

M.Taki, arXiv:0912.4789; arXiv:1007.2524;

Piotr Sulkowski, JHEP 1004 (2010) 063, arXiv:0912.5476,

N.Nekrasov and E.Witten, arXiv:1002.0888; 
R.Santachiara and A.Tanzini, arXiv:1002.5017;

S.Yanagida, arXiv:1003.1049; arXiv:1010.0528;

N.Drukker, D.Gaiotto and J.Gomis arXiv:1003.1112;

F.Passerini, JHEP 1003 (2010) 125, arXiv:1003.1151;

Wei He and Yan-Gang Miao, arXiv:1006.1214;

S.Kanno, Y.Matsuo and S.Shiba, arXiv:1007.0601;

H.Awata, H.Fuji, H.Kanno, M.Manabe and Y.Yamada, arXiv:1008.0574;

C.Kozcaz, S.Pasquetti, F.Passerini and N.Wyllard, arXiv:1008.1412;

H.Itoyama, T.Oota and N.Yonezawa, arXiv:1008.1861;

A.Braverman, B.Feigin, M.Finkelberg and L.Rybnikov, arXiv:1008.3655;

Ta-Sheng Tai, arXiv:1006.0471; arXiv:1008.4332;

M.Billo, L.Gallot, A.Lerda and I.Pesando, arXiv:1008.5240,

K.Maruyoshi and F.Yagi, arXiv:1009.5553;

A.Brini, M.Marino and S.Stevan, arXiv:1010.1210;

A.Mironov, A.Morozov and A.Shakirov, arXiv:1010.1734;

M.C.N.Cheng, R.Dijkgraaf adn C.Vafa, arXiv:1010.4573;

N.Wyllard, arXiv:1011.0289,

Y.Yamada, arXiv:1011.0292;

G.Bonelli, K.Maryoshi, A.Tanzini and F.Yagi, arXiv:1011.5417

[3] A.Marshakov, A.Mironov and A.Morozov, arXiv:0907.3946

[4] D.Gaiotto, arXiv:0908.0307;

A.Marshakov, A.Mironov and A.Morozov, Phys.Lett. B682 (2009) 125-129, arXiv:0909.2052;

V.Alba and And.Morozov, JETP Lett. 90 (2009) 708-712, arXiv:0911.0363

[5] A.Mironov and A.Morozov, Phys.Lett. B680 (2009) 188-194, arXiv:0908.2190

[6] A.Mironov and A.Morozov, Nucl.Phys. B825 (2009) 1-37, arXiv:0908.2569

[7] N.Nekrasov and S.Shatashvili, arXiv:0908.4052

[8] L.Alday, D.Gaiotto, S.Gukov, Y.Tachikawa and H.Verlinde, JHEP 1001 (2010) 113, arXiv:0909.0945

[9] A.Marshakov, A.Mironov and A.Morozov, JHEP 11 (2009) 048, arXiv:0909.3338

[10] A.Mironov and A.Morozov, Phys.Lett. B682 (2009) 118-124, arXiv:0909.3531

[11] R.Poghossian, JHEP 0912 (2009) 038, arXiv:0909.3412

[12] A.Mironov and A.Morozov, JHEP 04 (2010) 040, arXiv:0910.5670, J.Phys. A43 (2010) 195401, arXiv:0911.2396

A.Popolitov, arXiv:1001.1407

[13] H.Itoyama, K.Maruyoshi and T.Oota, Prog.Theor.Phys. 123 (2010) 957-987, arXiv:0911.4244

[14] T.Eguchi and K.Maruyoshi, arXiv:0911.4797

[15] R.Schiappa and N.Wyllard, arXiv:0911.5337

[16] A.Mironov, A.Morozov and Sh.Shakirov, JHEP 02 (2010) 030, arXiv:0911.5721

[17] V.Fateev and I.Litvinov, JHEP 1002 (2010) 014, arXiv:0912.0504

[18] A.Mironov, A.Morozov and Sh.Shakirov, arXiv:1001.0563 
[19] A.Mironov, A.Morozov and And.Morozov, arXiv:1003.5752

[20] H.Itoyama and T.Oota, arXiv:1003.2929

[21] C.Kozcaz, S.Pasquetti and N.Wyllard, arXiv:1004.2025

[22] T.Eguchi and K.Maruyoshi, arXiv:1006.0828

[23] K.Maruyoshi and M.Taki, arXiv:1006.4505

[24] A.Mironov, A.Morozov and A.Shakirov, arXiv:1011.3481

[25] A.Marshakov, A.Mironov and A.Morozov, arXiv:1011.4491

[26] A.Gerasimov, A.Marshakov, A.Mironov, A.Morozov and A.Orlov, Nucl.Phys. B357 (1991) $565-618$

[27] S.Kharchev, A.Marshakov, A.Mironov, A.Morozov and A.Zabrodin, Phys. Lett. B275 (1992) 311-314, hep-th/9111037; Nucl.Phys. B380 (1992) 181-240, hep-th/9201013;

S.Kharchev, A.Marshakov, A.Mironov and A.Morozov, Nucl.Phys. B397 (1993) 339-378, hep-th/9203043

[28] A.Mironov, A.Morozov and G.Semenoff, Int.J.Mod.Phys., A10 (1995) 2015, hep-th/9404005

A.Alexandrov, A.Mironov and A.Morozov, JHEP 12 (2009) 053, arXiv:0906.3305

[29] A.Morozov, Phys.Usp.(UFN) 35 (1992) 671-714; 37 (1994) 1, hep-th/9303139; hep-th/9502091; hep-th/0502010;

A.Mironov, Int.J.Mod.Phys. A9 (1994) 4355, hep-th/9312212; Phys.Part.Nucl. 33 (2002) $537 ;$ hep-th/9409190

[30] A.Gorsky, I.Krichever, A.Marshakov, A.Mironov, A.Morozov, Phys.Lett., B355 (1995) 466477, hep-th/9505035

[31] E.Martinec, Phys.Lett., B367 (1996) 91-96;

T. Nakatsu and K. Takasaki, Mod. Phys. Lett. A 11 (1996) 157 arXiv:hep-th/9509162;

R.Donagi and E.Witten, Nucl.Phys., B460 (1996) 299-334, hep-th/9510101

A.Gorsky, A.Marshakov, A.Mironov and A.Morozov, Phys.Lett., B380 (1996) 75-80, hep-th/9603140; hep-th/9604078;

A.Gorsky, S.Gukov and A.Mironov, Nucl.Phys., B517 (1998) 409-461; Nucl.Phys., B518 (1998) 689;

H.W.Braden, A.Marshakov, A.Mironov and A.Morozov, Nucl.Phys., B573 (2000) 553 hep-th/9906240; Phys.Lett., B448 (1999) 195, hep-th/9812078; Nucl.Phys., B573 (2000) 553, hep-th/9906240

A.Gorsky and A.Mironov, Nucl.Phys., B550 (1999) 513, hep-th/9902030; hep-th/0011197

A.Mironov and A.Morozov, hep-th/0001168

[32] E.Martinec and N.Warner, Nucl.Phys., 459 (1996) 97;

A.Gorsky, A.Marshakov, Phys.Lett., B374 (1996) 218-224;

H.Itoyama and A.Morozov, Nucl.Phys., B477 (1996) 855-877, hep-th/9511126; Nucl.Phys., B491 (1997) 529-573, hep-th/9512161, hep-th/9601168;

E.D'Hoker, I.M.Krichever and D.H.Phong, Nucl.Phys., B489 (1997) 179-210; Nucl.Phys., B489 (1997) 211-222;

N.Nekrasov, Nucl.Phys., B531 (1998) 323-344, hep-th/9609219

A.Marshakov, A.Mironov, Nucl.Phys., B518 (1998) 59-91;

A.Marshakov, Seiberg-Witten Theory and Integrable Systems, World Scientific, Singapore, 1999 
A.Mironov and A.Morozov, Phys.Lett., B475 (2000) 71;

H.Braden and A.Marshakov, Nucl.Phys. B595 (2001) 417-466; hep-th/0009060;

N.Nekrasov and S.Shatashvili, Nucl.Phys. Proc.Suppl. B192-193 (2009) 91-112, arXiv:0901.4744; arXiv:0901.4748

[33] A.Belavin, A.Polyakov, A.Zamolodchikov, Nucl.Phys. B241 (1984) 333-380;

A.Zamolodchikov and Al.Zamolodchikov, Conformal field theory and critical phenomena in 2d systems, 2009 (in Russian)

[34] N.Nekrasov, Adv.Theor.Math.Phys. 7 (2004) 831-864, hep-th/0206161;

N.Nekrasov and A.Okounkov, hep-th/0306238

[35] G.Moore, N.Nekrasov, S.Shatashvili, Nucl.Phys. B534 (1998) 549-611, hep-th/9711108; hep-th/9801061

A.Losev, N.Nekrasov and S.Shatashvili, Commun.Math.Phys. 209 (2000) 97-121, hep-th/9712241; ibid. 77-95, hep-th/9803265

[36] G.Moore and N.Seiberg, Comm.Math.Phys. 123 (1989) 177-254

[37] P.Argyres and A.Shapere, Nucl.Phys. B461 (1996) 437-459, hep-th/9509175

[38] N.Seiberg and E.Witten, Nucl.Phys., B426 (1994) 19-52, hep-th/9408099

[39] A.Alexandrov, A.Mironov and A.Morozov, Int.J.Mod.Phys. A19 (2004) 4127, hep-th/0310113

[40] A.Alexandrov, A.Mironov and A.Morozov, Int.J.Mod.Phys. A21 (2006) 2481-2518, hep-th/0412099 Fortsch.Phys. 53 (2005) 512-521, hep-th/0412205;

A.Alexandrov, A.Mironov, A.Morozov, P.Putrov, Int.J.Mod.Phys. A24 (2009) 4939-4998, arXiv:0811.2825

[41] A.Alexandrov, A.Mironov and A.Morozov, Teor.Mat.Fiz. 150 (2007) 179-192, hep-th/0605171; Physica D235 (2007) 126-167, hep-th/0608228; JHEP 12 (2009) 053, arXiv:0906.3305;

[42] B.Eynard, JHEP 0411 (2004) 031, hep-th/0407261;

L.Chekhov and B.Eynard, JHEP 0603 (2006) 014, hep-th/0504116 JHEP 0612 (2006) 026, math-ph/0604014;

N.Orantin, arXiv:0808.0635;

I.Kostov and N.Orantin, arXiv:1006.2028;

L.Chekhov, B.Eynard and O.Marchal, arXiv:1009.6007

[43] A.Gerasimov, S.Khoroshkin, D.Lebedev, A.Mironov and A.Morozov, Int.J.Mod.Phys. A10 (1995) 2589-2614, hep-th/9405011

A.Mironov, A.Morozov and L.Vinet, Theor.Math.Phys. 100 (1995) 890-899 (Teor.Mat.Fiz. 100 (1994) 119-131)

S.Kharchev, A.Mironov and A.Morozov, Theor.Math.Phys. 104 (1995) 129-143

A.Mironov, Theor.Math.Phys. 114 (1998) 127

A.Mironov and A.Morozov, Phys.Lett. B524 (2002) 217-226

[44] A.Marshakov, A.Mironov and A.Morozov, Phys. Lett. B389 (1996) 43; Mod.Phys.Lett. A12 (1997) 773-787; Int.J.Mod.Phys. A15 (2000) 1157-1206

[45] R.Flume and R.Poghossian, Int.J.Mod.Phys. A18 (2003) 2541, hep-th/0208176;

H.Nakajima and K.Yoshioka, math/0306198, math/0311058;

T.Eguchi and H.Kanno, JHEP 0312 (2003) 006, hep-th/0310235; Phys.Lett. B585 (2004) 163, hep-th/0312234 
J.Zhou, math.AG/0311237;

M.Taki, JHEP 0803 (2008) 048, arXiv:0710.1776

[46] A.Morozov and Sh.Shakirov, arXiv:0902.2627

[47] A.Alexandrov, arXiv:1009.4887

[48] F.David, Mod.Phys.Lett. A5 (1990) 1019;

A.Mironov and A.Morozov, Phys.Lett. B252 (1990) 47-52;

J.Ambjorn and Yu.Makeenko, Mod.Phys.Lett. A5 (1990) 1753;

H.Itoyama, Y.Matsuo, Phys.Lett., 255B (1991) 202

[49] J.Harer and D.Zagier, Invent.Math. 85 (1986) 457-485;

S.K.Lando and A.K.Zvonkin, Embedded graphs, Max-Plank-Institut fr̈ Mathematik, Preprint Series 2001 (63)

[50] A.Morozov and Sh.Shakirov, arXiv:0906.003

[51] A.Morozov and Sh.Shakirov, arXiv:1007.4100

[52] F.Götze and A.Tikhomirov, Cent.Eur.J.Math. 3 (2005) 666-704;

U.Haagerup and S.Thorbjørnsen, arXiv:1004.3479

[53] L.Chekhov, A.Marshakov, A.Mironov and D.Vasiliev, Phys.Lett. B562 (2003) 323, hep-th/0301071; Proc. Steklov Inst.Math. 251 (2005) 254, hep-th/0506075

[54] A.Morozov and Sh.Shakirov, JHEP 0904 (20090 064, arXiv:0902.2627;

A.Alexandrov, arXiv:1005.5715;

A.Morozov, Theor.Math.Phys. 162 (2010) 1-33 (Teor.Mat.Fiz. 161 (2010) 3-40), arXiv:0906.3518;

A.Balantekin, arXiv:1011.3859;

A.Alexandrov, A.Mironov and A.Morozov, Cut-and-Join Operators, Matrix Models and Characters, to appear

[55] M. Kontsevich, Funk.Anal.Prilozh., 25:2 (1991) 50-57; Comm.Math.Phys. 147 (1992) 1-23

[56] A.Marshakov, A.Mironov, and A.Morozov, Phys.Lett. B265 (1991) 99

S.Kharchev, A.Marshakov, A.Mironov, A.Morozov and S.Pakuliak, Nucl.Phys. B404 (1993) 17-750, arXiv:hep-th/9208044

[57] L.Chekhov and A.Mironov, Phys.Lett. B552 (2003) 293, hep-th/0209085;

V.Kazakov and A.Marshakov, J.Phys. A36 (2003) 3107, hep-th/0211236;

A.Mironov, Theor.Math.Phys. 146 (2006) 63-72, hep-th/0506158;

B.Eynard and N.Orantin, arXiv:math-ph/0702045

[58] E.T.Whittaker and G.N.Watson, A Course of Modern Analysis, Cambridge University Press

[59] I.S.Gradshteyn and I.M.Ryzhik, Table of Integrals, Series, and Products, Academic Press

[60] N.Seiberg and E.Witten, Nucl.Phys., B431 (1994) 484-550, hep-th/9407087

[61] Al.Zamolodchikov, JETP 63 (1986) 1061; Theor.Math.Phys. 73 (1987) 1088; Nucl.Phys. B285 (1987) 481

[62] V.Fateev and A.Litvinov, JHEP 0711 (2007) 002, arXiv:0709.3806

[63] A.Braverman, arXiv:math/0401409

A.Braverman and P.Etingof, arXiv:math/0409441 